\title{
FXR LIA Optimization \\ Time-resolved OTR Emittance Measurement
}

\author{
J.S. Jacob, M.M. Ong, P. Wargo, G.P. LeSage
}

October 6, 2005 


\section{Disclaimer}

This document was prepared as an account of work sponsored by an agency of the United States Government. Neither the United States Government nor the University of California nor any of their employees, makes any warranty, express or implied, or assumes any legal liability or responsibility for the accuracy, completeness, or usefulness of any information, apparatus, product, or process disclosed, or represents that its use would not infringe privately owned rights. Reference herein to any specific commercial product, process, or service by trade name, trademark, manufacturer, or otherwise, does not necessarily constitute or imply its endorsement, recommendation, or favoring by the United States Government or the University of California. The views and opinions of authors expressed herein do not necessarily state or reflect those of the United States Government or the University of California, and shall not be used for advertising or product endorsement purposes.

This work was performed under the auspices of the U.S. Department of Energy by University of California, Lawrence Livermore National Laboratory under Contract W7405-Eng-48. 
Table of Contents

\section{FXR LIA OPTIMIZATION - TIME-RESOLVED OTR EMITTANCE MEASUREMENT}

Abstract


Table of Figures

Table 1.1. Measured emittance at high-current linear induction accelerators............................3

Figure 2.1. Relationship of the Twiss parameters to the trace space distribution. .......................7

Figure 2.2. Evolution of the trace space distribution as the beam goes through a focus ...............7

Table 2.1. Comparison of spot size and emittance values for various beam distributions ............8

Figure 2.3. Final spot size as a function of emittance, energy variation, focal length, and entering

beam radius.

Figure 3.1. Angular dependence of the spectral intensity per unit solid angle at $4.5 \mathrm{MeV}$ and 20

$\mathrm{MeV}$.....

Figure 3.2. Image charge representation of back-emitted transition radiation...........................13

Figure 3.3. Theoretically predicted OTR angular distribution from a single electron................14

Figure 3.4. Definition of polarization directions .........................................................14

Figure 3.5. Lineouts of the OTR angular pattern in the parallel and perpendicular directions for a

single electron at $17.5 \mathrm{MeV}$

Figure 3.6. Comparison of OTR angular pattern from single electron at $17.5 \mathrm{MeV}$ with beam at

$17.5 \mathrm{MeV}$ and $15 \mathrm{mrad}$ RMS divergence....

Figure 3.7. Vertical lineout of OTR angular pattern for a single electron and a beam with 15

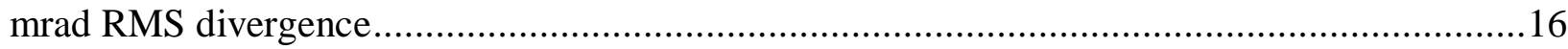

Figure 3.8. Comparison of valley-to-peak ratio for different beam distributions .......................17

Figure 3.9. OTR angular pattern at different wavelengths......................................... 17

Figure 3.10. OTR angular pattern vs. energy variation................................................ 18

Table 4.1. Thermal properties and minimum tolerable spot size for various target materials ......19

Figure 5.1. Simultaneous observation of beam divergence and spatial profile ..........................20

Figure 5.2. Raytrace simulation of angular data collection optics using OSLO.....................21

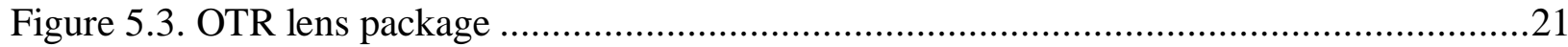

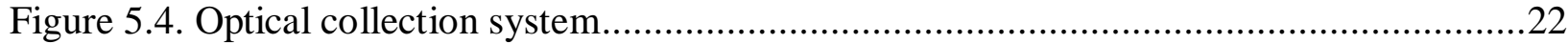

Figure 5.5. Raw data images in WinView32 software....................................................2

Figure 5.6. Spot size calculation software from Greg LeSage and Scott Anderson...................23

Figure 5.7. Image processing LabVIEW VI for OTR angular data........................................24

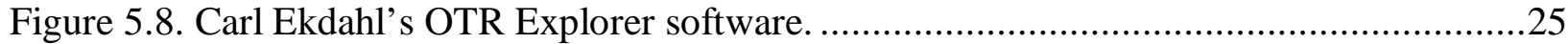

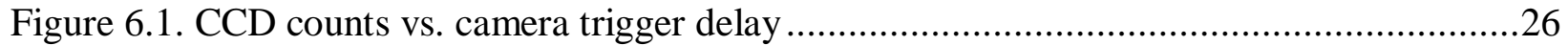

Figure 6.2. Beam pulse current measurement ................................................................26

Figure 6.3. RMS spot size as a function of DR2 current .................................................27

Figure 6.4. Beam profile images at 60, 80, and $100 \mathrm{~ns}$ camera trigger delay. .........................27

Figure 6.5. Spot size analysis showing experimental data from FXR and best Gaussian fit .......28

Figure 6.6. Angular distribution images at 60, 80, and $100 \mathrm{~ns}$ camera trigger delay .................28

Figure 6.7. Horizontal lineout of OTR angular distribution............................................29

Figure 6.8. Least-squares fit in OTR Explorer software ......................................................30

Figure 6.9. RMS spot size, RMS divergence, and RMS emittance as a function of time. ...........30

Table 7.1. Summary of recent emittance measurements at FXR ........................................31 


\title{
FXR LIA Optimization - Time-resolved OTR Emittance Measurement
}

\author{
J. S. Jacob, M. M. Ong, G. P. LeSage \\ Lawrence Livermore National Laboratory, 7000 East Ave. L-153, \\ Livermore, CA, 94551 \\ P. Wargo \\ Bechtel Nevada, 161 S. Vasco Rd. Suite A, Livermore, CA 94551
}

\begin{abstract}
The Flash X-Ray Radiography (FXR) facility at Lawrence Livermore National Laboratory utilizes a high current, long pulse linear induction accelerator to produce high doses of X-ray radiation. Accurate characterization of the transverse beam emittance is required in order to facilitate accelerator modeling and tuning efforts and, ultimately, to optimize the final focus spot size, yielding higher resolution radiographs. In addition to conventional magnet scan, pepper-pot, and multiple screen techniques, optical transition radiation (OTR) has been proven as a useful emittance measurement diagnostic and is particularly well suited to the FXR accelerator. We shall discuss the time-resolved emittance characterization of an induction linac electron beam using OTR, and we will present our experimental apparatus and analysis software. We shall also develop the theoretical background of beam emittance and transition radiation.
\end{abstract}

\section{INTRODUCTION}

The LLNL Flash X-Ray Radiography (FXR) facility is capable of producing high doses ( $10 \mathrm{R})$ of x-ray radiation by focusing a $3 \mathrm{kA}, 70 \mathrm{~ns}, 17.5 \mathrm{MeV}$ electron beam into a Tantalum bremsstrahlung target [1]. In order to improve the spatial resolution of FXR radiographs, a considerable effort has been undertaken to identify and improve key factors limiting the final focus spot size of the FXR accelerator. Transverse beam emittance, energy variation, beam motion, and focusing aberrations can all play a role in limiting the electron beam focus [2]. At FXR, emittance and energy variation have been identified as the key limiting factors. In this paper, we shall focus primarily on efforts to characterize and reduce the emittance of the FXR injector and accelerator.

Transverse emittance is a measure of the phase space volume of the beam in the dimensions perpendicular to the beam axis. Qualitatively, emittance can be thought of as the temperature of the beam -- a measure of the random disorder in the transverse momenta of the constituent particles. Quantitatively, transverse emittance is defined by drawing a contour around a given percentage of particles in phase space and gives us a numerical figure of merit for describing the quality of the beam. As the beam is focused or defocused, the convergence or divergence of the beam envelope yields a correlation between particle position and angle of motion; if, however, we focus the beam to a waist, the correlation between particle position and transverse momentum is minimized. The remaining random angular spread of the beam, together with the radial profile, characterizes the emittance [3]. We can easily imagine that a perfectly "cold" beam with zero emittance could be focused to an infinitely small point by a perfectly linear focusing lens (ignoring space charge effects). The random angular spread of a beam with nonzero emittance, 
however, makes this impossible - the larger the emittance, the larger the random angular motion of the particles, and thus the larger the final focus spot.

A variety of emittance measurement techniques are employed on particle accelerators today, including collimation of the beam with an array of small apertures ("pepper-pot" technique) [4], magnet scan techniques in which the beam profile is characterized as a function of varying focusing parameters [5], and multi-profile techniques in which the beam profile is characterized simultaneously at several locations [6]. Optical Transition Radiation (OTR) provides an attractive alternative to these methods and avoids many of the potential pitfalls that make other measurement techniques difficult to implement on a high-current, high-energy beam -- magnet scan techniques are influenced by space charge effects (especially with solenoid focusing magnets), and the high energy at the exit of the accelerator requires pepper-pot grids of an unreasonable thickness to effectively stop the beam. With the right equipment, OTR allows emittance to be measured at a single point in a single shot without varying the beam envelope or attenuating a significant portion of the beam. Time-resolved measurement of emittance is possible with fast camera gating, and spatially resolved measurements in selected parts of the beam profile can be achieved through clever target foil design [7].

The existence of transition radiation was predicted by Ginzburg and Frank in 1946 [8] and the theory was thoroughly developed throughout the 1970s [9-12]. From 1975 through the present day, OTR has been utilized for beam characterization on high-energy particle accelerators [13$16]$ and, in particular, on high-current induction linacs [7, 17]. Transition radiation is emitted as a moving charged particle crosses the boundary between two media of differing dielectric properties, typically vacuum and a thin metallic foil in the case of a beam diagnostic. Although TR is inherently broadband, a significant portion of the light is emitted across the visible spectrum for relativistic electron beams [18], hence the term optical transition radiation. Because of this, we can utilize conventional optics and cameras designed for visible light. OTR light is emitted in a characteristic angular distribution that depends on the energy and incident angle of the particle. Since an electron beam is an ensemble of many particles with differing energies and angles of motion, the observed pattern is a "blurred" superposition of many single-particle patterns. By empirically measuring the "blur" of the pattern and fitting to a theoretically predicted distribution, we can deduce the angular spread of the beam and, ultimately, the emittance.

Emittance is a conserved quantity - although nonlinear focusing and accelerating forces can cause an effective growth of emittance through the accelerator, angular motion that is not correlated to position in the beam cannot be later reduced. Thus, it is critical to tune the injector to generate a beam with the lowest possible emittance in order to produce the best possible final focus. Pepper-pot measurements by T. Houck et. al. [24] indicate that the normalized emittance is $193 \mathrm{~cm}-\mathrm{mr}$ at the FXR injector exit, which is both considerably larger than simulations predict (36 cm-mr) and also much larger than measured emittance at other similar facilities $(75 \mathrm{~cm}-\mathrm{mr}$ at DARHT-I, $47 \mathrm{~cm}-\mathrm{mr}$ at ETA-II, and $110 \mathrm{~cm}-\mathrm{mr}$ at ARIX). Measurements at the exit of the accelerator yield a normalized emittance of $385 \mathrm{~cm}$-mr on average, indicating emittance growth through the linac and highlighting the need for further diagnosis and modeling. 


\begin{tabular}{|l|l|l|c|l|c|c|}
\hline Accelerator & $\begin{array}{c}\text { Cathode } \\
\text { Type }\end{array}$ & $\begin{array}{c}\text { Energy } \\
(\mathrm{MeV})\end{array}$ & $\begin{array}{c}\text { Current } \\
(\mathrm{kA})\end{array}$ & $\begin{array}{c}\text { Type } \\
\text { Measurement }\end{array}$ & $\begin{array}{c}\text { Normalized } \\
\varepsilon_{N, r m s}(\mathrm{~cm}-\mathrm{mr})\end{array}$ & $\begin{array}{c}\text { Scaled } \\
\varepsilon_{N, r m s}(\mathrm{~cm}-\mathrm{mr})\end{array}$ \\
\hline ARIX & Velvet & 3.85 & 2.0 & Envelope & 110 & 115 \\
\hline ETA-II $^{8}$ & Dispenser & 2.55 & 1.4 & Pepper Pot & 61 & 93 \\
\hline ETA-II $^{9}$ & Dispenser & 6.0 & 2.0 & Pepper Pot & 47 & 60 \\
\hline DARHT-I $^{10}$ & Velvet & 3.75 & 4.0 & Envelope & 75 & 67 \\
\hline FXR $^{11}$ & Velvet & 17.5 & 3.3 & OTR & 370 & 370 \\
\hline FXR $^{12}$ & Velvet & 17.9 & 3.3 & Envelope & 400 & 400 \\
\hline FXR $^{13}$ & Velvet & 2.1 & 3.3 & Pepper Pot & 193 & 193 \\
\hline FXR & Velvet & 2.1 & 3.3 & Simulation & 36 & 36 \\
\hline
\end{tabular}

Table 1.1. Measured emittance at high-current linear induction accelerators. From T. Houck [24].

Recent modeling efforts have confirmed the robustness of the FXR injector design with regards to mechanical alignment and position perturbations as well as small errors in the magnetic fields and, in general, suggest that the current injector design should produce a much lower emittance than is observed [24]. Three different codes (TRAK, OmniTrak, and LSP) were utilized to simulate the FXR injector and, although they agree well with each other, they clearly do not agree well with reality, suggesting that some feature of the underlying physics is not being correctly modeled. A likely explanation for this discrepancy is that the emission from the cathode is not uniform - simulations show that a large (10\% of total area) non-emitting region at the center of the cathode could double the emittance, and imaging of the plasma light generated at the cathode displays a non-uniform, mottled appearance which is, unfortunately, beyond current modeling capabilities to simulate [24]. A possible solution may be to redesign the injector to increase the electric field gradient on the cathode, leading to more uniform emission; since the details of the current distribution cannot be accurately modeled, empirical emittance measurements are the only option for evaluating the effects of a redesign on injector emittance. Another possible explanation for the higher than expected emittance is the interaction of the beam with the metallic pepper-pot foil - simulations show that the presence of a thin metallic foil at the injector exit doubles the emittance of the beam [24]. Together, these two effects may account for the discrepancy between predicted and measured emittance values. In order to test the efficacy of new tunes and hardware improvements on beam emittance, a robust, reliable, and fast measure of beam emittance must be available to provide empirical feedback on theoretical modeling efforts; it is with this goal in mind that the OTR emittance measurement diagnostic has been developed and implemented on FXR.

\section{BEAM PHYSICS}

\section{Emittance}

In order to establish the theoretical background for beam emittance, we shall follow a treatment by B. M. van Oerle [19]. The motion of an ensemble of free electrons can be completely described by a six-dimensional space called "phase space" spanned by the three spatial coordinates and the three momentum components of the particles. In general, we need not deal with the entire six-dimensional space at once; in particular, if we can define a coordinate system such that there is no correlation between the $\mathrm{x}, \mathrm{y}$, and $\mathrm{z}$ dimensions, we can isolate each 
dimension into its own two-dimensional space called "trace space." Let us define a Cartesian coordinate system in which the z-axis is the longitudinal axis of the beam. The trace space for the $\mathrm{x}$ dimension is spanned by the spatial coordinate $\mathrm{x}$ and the inclination of the velocity vector,

$$
x^{\prime} \equiv \frac{p_{x}}{p_{z}}
$$

. A similar description applies for the $\mathrm{y}$ dimension. Together, the $\mathrm{x}$ and $\mathrm{y}$ trace spaces define the "transverse" emittance of the beam, while the $\mathrm{z}$ dimension defines the "longitudinal" emittance. Here, we shall limit our discussion to the transverse emittance.

The mean value of an arbitrary quantity A over the particle distribution is given by

$$
\langle A\rangle=\iint f\left(x, x^{\prime}\right) A d x d x^{\prime}
$$

, where $f\left(x, x^{\prime}\right)$ describes the distribution of electrons in trace space and can be Gaussian, parabolic, uniform, or completely arbitrary in form. The distribution function is normalized such that the integral of $\mathrm{f}\left(\mathrm{x}, \mathrm{x}^{\prime}\right)$ over all trace space gives the number of particles in the distribution. We can now define the RMS values for the spatial width and angular spread of the distribution:

$$
\begin{aligned}
& x_{r m s} \equiv \sqrt{\left\langle x^{2}\right\rangle} \\
& x_{r m s}^{\prime} \equiv \sqrt{\left\langle x^{\prime 2}\right\rangle}
\end{aligned}
$$

In order to fully describe the evolution of the particle beam, we must understand the evolution of the second moments of the distribution: $\left\langle x x^{\prime}\right\rangle,\langle x x\rangle$, and $\left\langle x^{\prime} x^{\prime}\right\rangle$. The time derivative of $\left\langle x x^{\prime}\right\rangle$ is given by

$$
\begin{aligned}
\frac{d}{d t}\left\langle x x^{\prime}\right\rangle & =\iint\left[\frac{\partial f\left(x, x^{\prime}\right)}{\partial t} x x^{\prime}+f\left(x, x^{\prime}\right) \frac{d x}{d t} x^{\prime}+f\left(x, x^{\prime}\right) x \frac{d x^{\prime}}{d t}\right] d x d x^{\prime} \\
& =\iint \frac{\partial f\left(x, x^{\prime}\right)}{\partial t} x x^{\prime} d x d x^{\prime}+\left\langle x \frac{d x^{\prime}}{d t}\right\rangle+\left\langle\frac{d x}{d t} x^{\prime}\right\rangle
\end{aligned}
$$

If we assume the distribution is not explicitly dependent on time, $\frac{\partial f}{\partial t}=0$, so

$$
\frac{d}{d t}\left\langle x x^{\prime}\right\rangle=\left\langle x \frac{d x^{\prime}}{d t}\right\rangle+\left\langle\frac{d x}{d t} x^{\prime}\right\rangle
$$

and 


$$
\begin{aligned}
& \frac{d}{d t}\langle x x\rangle=2\left\langle x \frac{d x}{d t}\right\rangle \\
& \frac{d}{d t}\left\langle x^{\prime} x^{\prime}\right\rangle=2\left\langle x^{\prime} \frac{d x^{\prime}}{d t}\right\rangle
\end{aligned}
$$

We can define an equation of motion for the ensemble of particles by recognizing that each particle experiences two categories of force: external transport forces $F_{e}$ and self force $F_{s}$ from interactions with the other particles in the ensemble. Thus, for each particle,

$$
m \frac{d^{2} x^{i}}{d t^{2}}=F_{s}^{i}+F_{e}^{i}
$$

, where $F_{s}=\sum_{j \neq i} F_{s}^{i, j}$. If we substitute this equation of motion into equations 2.6-2.8, we obtain

$$
\begin{aligned}
& \frac{d}{d t}\left\langle x x^{\prime}\right\rangle=\frac{1}{p_{z}}\left\langle x\left(F_{s}+F_{e}\right)\right\rangle+\frac{p_{z}}{m}\left\langle x^{\prime 2}\right\rangle \\
& \frac{d}{d t}\left\langle x^{\prime 2}\right\rangle=\frac{2}{p_{z}}\left\langle x^{\prime}\left(F_{s}+F_{e}\right)\right\rangle \\
& \frac{d}{d t}\left\langle x^{2}\right\rangle=\frac{2 p_{z}}{m}\left\langle x x^{\prime}\right\rangle
\end{aligned}
$$

We now define the $\sigma$-matrix,

$$
\sigma \equiv\left(\begin{array}{ll}
\left\langle x^{2}\right\rangle & \left\langle x x^{\prime}\right\rangle \\
\left\langle x x^{\prime}\right\rangle & \left\langle x^{\prime 2}\right\rangle
\end{array}\right)
$$

In order to simplify our analysis, we shall make some assumptions regarding the nature of the forces acting on the particles. First, since accelerator transport optics are generally carefully designed to ensure linearity in order to prevent emittance growth, we shall assume that the transport forces are linear in $\mathrm{x}$, that is, $F_{e}(t)=-k(t) x$. We shall also assume that the self-force of the particles is linearly dependent on $\mathrm{x}$ such that $\left\langle x F_{s}\right\rangle=\alpha\left\langle x^{2}\right\rangle$. We can now express equations 2.10-2.12 as the transformation of the $\sigma$-matrix:

$$
\frac{d}{d t} \sigma=F \cdot \sigma+\sigma \cdot F^{T}
$$


, where

$$
F=\left(\begin{array}{cc}
0 & \frac{p_{z}}{m} \\
\frac{-k+\alpha}{p_{z}} & 0
\end{array}\right)
$$

In the special case that the self-force of the particles can be neglected ( $\alpha \approx 0$ ), our analysis can be further simplified:

$$
\sigma_{\text {final }}=R \cdot \sigma_{\text {initial }} \cdot R^{T}
$$

. In this case, we say that the beam is "emittance dominated." We should note that, although space charge effects are not entirely negligible, simulations indicate that the FXR beam is emittance dominated at the exit of the accelerator [25, p. 24]. $\mathrm{R}$ is called the transport matrix and is defined as

$$
R=I+\int_{t_{i}}^{t_{f}} F(t) d t
$$

, where I is the identity matrix. The transport matrix also gives us the evolution of the first moments of the distribution:

$$
\left(\begin{array}{c}
\left\langle x_{f}\right\rangle \\
\left\langle x_{f}^{\prime}\right\rangle
\end{array}\right)=R \cdot\left(\begin{array}{c}
\left\langle x_{i}\right\rangle \\
\left\langle x_{i}^{\prime}\right\rangle
\end{array}\right)
$$

Emittance is defined as the area in trace space enclosed by a contour that encompasses a given number of particles in the distribution. We define the RMS emittance as the square root of the determinant of the $\sigma$-matrix:

$$
\varepsilon_{x} \equiv \sqrt{|\sigma|}=\sqrt{\left.\left\langle x^{2}\right\rangle\left\langle x^{\prime 2}\right\rangle-<x x^{\prime}\right\rangle^{2}}
$$

The second moment term $\left\langle x x^{\prime}\right\rangle^{2}$ represents a correlation between $\mathrm{x}$ and $\mathrm{x}$ ' that exists when the beam envelope is converging or diverging; qualitatively, it can be thought of as a measure of inward or outward flow of transverse kinetic energy. At a waist, this correlation is minimized and the second moment term is zero [22]. Thus, at a waist, the emittance reduces to

$$
\varepsilon_{x}=\sqrt{\left\langle x^{2}\right\rangle\left\langle x^{\prime 2}\right\rangle}=x_{r m s} x_{r m s}^{\prime}
$$


This is particularly useful for our diagnostic purposes, since the RMS spot size and divergence can be independently measured to yield the emittance.

In order to more clearly express the relationship between emittance and trace space, we can rewrite the $\sigma$-matrix as

$$
\sigma=\varepsilon\left(\begin{array}{cc}
\beta^{\prime} & -\alpha^{\prime} \\
-\alpha^{\prime} & \gamma^{\prime}
\end{array}\right)
$$

. In this form, $\alpha^{\prime}, \beta^{\prime}$, and $\gamma^{\prime}$ are called Twiss or Courant-Snyder parameters, and they obey the Courant-Snyder equality $\beta^{\prime} \gamma^{\prime}-a^{\prime 2}=1$. Thus, the ellipse in trace space has the equation

$$
\gamma^{\prime} x^{2}+2 \alpha^{\prime} x x^{\prime}+\beta^{\prime} x^{\prime 2}=\varepsilon
$$

and has area equal to pi times the emittance. The figures below illustrate the relationship of the Twiss parameters to the trace space ellipse and show the evolution of the trace space distribution as the beam goes through a focus.

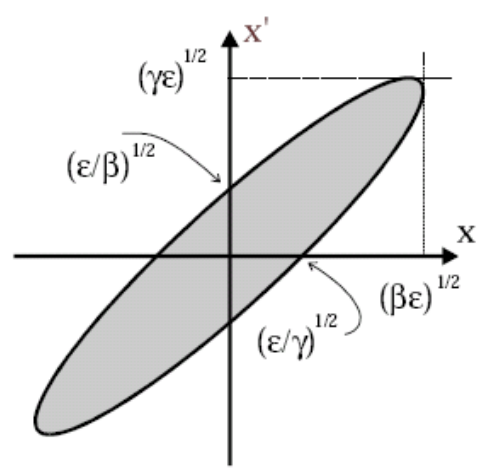

Figure 2.1. Relationship of the Twiss parameters to the trace space distribution. From B.M van Oerle [19].


Figure 2.2. Evolution of the trace space distribution as the beam goes through a focus. From B.M. van Oerle [19].

If we multiply the above definition of emittance by $\beta \gamma$ (the normalized velocity and the relativistic Lorentz factor of the electron bunch), we obtain the normalized emittance, 


$$
\varepsilon_{\text {norm }}=\beta \gamma \varepsilon
$$

. The normalized emittance is invariant under changes of energy, an obvious benefit when dealing with accelerators. Note that emittance is conserved only if the assumptions made in our analysis above hold true - nonlinear accelerating and transport forces and space charge effects can all cause an effective emittance growth.

Our chosen definition of RMS emittance is by no means the only valid description, a fact that is made painfully obvious by the lack of a universally consistent definition in the literature - beam physicists from different institutions often utilize their own unique definition of emittance. For trace space distributions with a clearly defined boundary or edge, it is often useful to refer to the "edge emittance" or the " $100 \%$ emittance." In this case, the trace space ellipse is chosen to encompass all (or most) of the particles in the distribution. Edge emittance is also a particularly attractive choice when dealing with extremely high power beams or beams of massive particles in which the loss of even a small portion of the beam is undesirable; FXR falls into this category, and so edge values are frequently utilized. The relationship between edge and RMS emittance is not necessarily straightforward and depends on the particular beam distribution. The table below was produced by Art Paul [20] and provides conversion factors for a number of common distributions.

\begin{tabular}{|c|c|c|c|c|c|c|}
\hline $\begin{array}{c}\text { Distribution } \\
\text { function }\end{array}$ & $\begin{array}{c}\text { cutoff } \\
\text { amplitude }\end{array}$ & $\begin{array}{l}\text { beam } \\
\text { edge }\end{array}$ & $r_{\mathrm{mas}}$ & $\mathrm{r}_{\mathrm{h}}$ & $\varepsilon_{\mathrm{mas}}$ & $\varepsilon_{\mathrm{he}}$ \\
\hline uniform & - & $\mathrm{a}$ & $a / \sqrt{2}$ & $\mathrm{a}$ & $1 / 2 a^{\prime}$ & $2 \varepsilon_{\mathrm{nms}}$ \\
\hline parabolic & - & a & $a / \sqrt{3}$ & $a \sqrt{2}$ & $1 / 3 a^{\prime}$ & $3 \varepsilon_{\mathrm{mms}}$ \\
\hline Gaussian & - & $\infty$ & $\sigma$ & $\sqrt{\ln 2} \sigma$ & $\sigma \sigma^{\prime}$ & - \\
\hline$"$ & 0.13534 & $\sqrt{2}$ & - & - & - & $2 \varepsilon_{\mathrm{ms}}$ \\
\hline$"$ & 0.1 & $\sqrt{\ln 10} \sigma$ & - & - & - & 2. $30 \varepsilon_{\mathrm{mms}}$ \\
\hline$"$ & 0.0498 & $\sqrt{3} \sigma$ & - & - & - & $3 \varepsilon_{\mathrm{ms}}$ \\
\hline " & 0.0183 & $2 \sigma$ & - & - & - & $4 \varepsilon_{\mathrm{ms}}$ \\
\hline
\end{tabular}

Table 2.1. Comparison of spot size and emittance values for various beam distributions. From Art Paul [20].

\section{Spot size}

Now that we have defined emittance, we would like to know its effect on our ability to focus the beam. We shall follow a treatment by Yu-Jiuan Chen relating final focus spot size to emittance, energy variation, and other beam parameters for a solenoid focusing system such as that found on FXR [21]. Throughout our analysis, we shall use the subscripts 0 and $f$ to denote conditions at the entrance of the focusing lens and at the focal point, respectively. We shall also utilize the thin-lens approximation and assume that our focusing lens changes only particle trajectory and not particle location over the length of the lens; in this case, spot size at the entrance and exit of the lens are equal. We begin with the Lee-Cooper's RMS envelope equation for an emittancedominated, coasting beam:

$$
R^{\prime \prime}=\frac{\varepsilon^{2}}{R^{3}}
$$


, where $\mathrm{R}$ is the RMS spot size and $\varepsilon$ is the RMS emittance, as defined above. As noted in the previous section, simulations indicate that the FXR beam is emittance-dominated at the exit of the accelerator [25, p. 24]. Multiplying by R' and integrating both sides, we obtain

$$
R^{\prime 2}-R_{0}^{\prime 2}=\frac{\varepsilon^{2}}{R_{0}{ }^{2}}-\frac{\varepsilon^{2}}{R^{2}}
$$

At the focal point, $\mathrm{R}_{\mathrm{f}}{ }^{\prime}=0$, so we can write the above equation as

$$
R_{f}{ }^{2}=\frac{\varepsilon^{2} R_{0}{ }^{2}}{R_{0}{ }^{2} R_{0}^{\prime 2}+\varepsilon^{2}} \approx\left(\frac{\varepsilon}{\left|R_{0}^{\prime}\right|}\right)^{2}=\left(\frac{\varepsilon f}{R_{0}}\right)^{2}
$$

, where

$$
f=\frac{R_{0}}{\left|R_{0}^{\prime}\right|}
$$

. The final approximation above assumes that $R_{0}\left|R_{0}^{\prime}\right| \gg \mathcal{E}$ since the final spot size is usually much less than the spot size at the entrance to the focusing lens. Thus,

$$
R_{f} \approx \frac{\varepsilon f}{R_{0}}
$$

As we mentioned in the introduction, energy variation as well as emittance limits the final focus spot size at FXR, since energy spread leads to chromatic aberration in the final focus system. To investigate the effect of energy spread on spot size, we shall follow an analysis by M. Reiser [22, p. 108]. Since the focal length of the lens depends on the momentum of the particles, particles with different momenta are imaged to different focal planes downstream of the lens; thus, if we consider the entire ensemble of momenta and trajectories within the beam, we can imagine that the beam will not be focused to a single point, but rather to a waist of non-zero radius which, in optics terminology, is commonly known as the "circle of least confusion." We shall refer to the radius of this circle as $R_{c}$. In order to simplify our analysis, we will assume that the beam is collimated at the entrance to the final focus lens, that is, $R_{0}{ }^{\prime}=0$. The spread in image locations, $\Delta \mathrm{z}_{\mathrm{i}}$, depends on the momentum spread, $\Delta \mathrm{P}$. If we consider $\mathrm{P}$ to be the average momentum of particles in the beam, then particles with momentum $\mathrm{P}+\Delta \mathrm{P}$ will be focused at a point $\mathrm{z}_{\mathrm{f}}+\Delta \mathrm{z}_{\mathrm{f}}$, where

$$
\Delta z_{f}=\left(\frac{\partial f}{\partial p}\right) \Delta P
$$

If the angle of convergence for the particle with momentum $\mathrm{P}$ is $\alpha$, then 


$$
R_{c}=\alpha\left(\frac{\partial f}{\partial P}\right) \Delta P=\alpha f\left(\frac{P}{f} \frac{\partial f}{\partial P}\right) \frac{\Delta P}{P}
$$

We define the chromatic aberration coefficient, $\mathrm{C}_{\mathrm{c}}$, as

$$
\frac{C_{c}}{f}=\frac{1}{2}\left(\frac{P}{f} \frac{\partial f}{\partial P}\right)
$$

For a thin solenoidal lens, this coefficient is simply equivalent to the focal length of the lens: $\mathrm{C}_{\mathrm{c}}=\mathrm{f}[22, \mathrm{p} .109]$. Thus, we find that

$$
R_{c}=2 \alpha C_{c} \frac{\Delta P}{P}=2 \alpha f \frac{\Delta P}{P}=2 \alpha f \frac{\Delta \gamma}{\beta^{2} \gamma}
$$

Finally, by noting that $R_{0}=f \cdot \tan (\alpha) \approx \alpha f$, we obtain

$$
R_{c}=2 R_{0} \frac{\Delta \gamma}{\beta^{2} \gamma}
$$

This gives us the minimum achievable spot size if chromatic aberration were the only limiting factor. Combined with emittance, the two effects add in quadrature, and so the overall expression for our spot size is

$$
R_{f}^{2}=\left(\frac{\varepsilon f}{R_{0}}\right)^{2}+\left(2 R_{0} \frac{\Delta \gamma}{\beta^{2} \gamma}\right)^{2}
$$

The chart below illustrates the combined effects of emittance, energy variation, and beam size at the focus magnet entrance on the final focus spot size. We should note that although every lens has some inherent spherical aberration, the effect is small compared to emittance (a few percent for a nominal FXR beam), and so we have chosen to ignore this effect in our discussion [21]. 




Figure 2.3. Final spot size as a function of emittance, energy variation, focal length, and entering beam radius. From Y. Chen [21].

\section{OTR THEORY}

Transition radiation is emitted whenever a charged particle crosses the boundary between two media of differing dielectric properties. Qualitatively, we can understand the effect by recognizing that a moving charged particle carries an electric field with it, which depends on the dielectric constant, $\varepsilon$, of the medium. As the particle crosses into the new medium, the fields must reorganize themselves in the presence of the new dielectric constant and, in the process, some of the fields are "shaken off" as transition radiation [18].

A rigorous analytical treatment of transition radiation involves using Maxwell's equations to solve for the radiation fields in the two media and applying the proper boundary conditions. The most general case is an electron passing from medium 1 with dielectric constant $\varepsilon_{1}$ into medium 2 with dielectric constant $\varepsilon_{2}$ at normal incidence to the boundary. The expression for the spectral intensity, I, of the radiation emitted into medium 2 into the solid angle $\mathrm{d} \Omega$ and in frequency range $\mathrm{d} \omega$ was derived by Ter-Mikaelian [10]:

$$
\begin{aligned}
& \frac{d^{2} I_{2}(\theta, \omega)}{d \omega d \Omega}= \\
& \frac{e^{2} \beta^{2} \sqrt{\varepsilon_{2}} \sin ^{2} \theta_{2} \cos ^{2} \theta_{2}}{\pi^{2} c} \\
& \times\left|\frac{\left(\varepsilon_{1}-\varepsilon_{2}\right)\left(1-\beta^{2} \varepsilon_{2}-\beta \sqrt{\varepsilon_{1}-\varepsilon_{2} \sin ^{2} \theta_{2}}\right)}{\left(1-\beta^{2} \varepsilon_{2} \cos ^{2} \theta_{2}\right)\left(1-\beta \sqrt{\varepsilon_{1}-\varepsilon_{2} \sin ^{2} \theta_{2}}\right)\left(\varepsilon_{1} \cos \theta_{2}+\sqrt{\varepsilon_{1} \varepsilon_{2}-\varepsilon_{2}^{2} \sin ^{2} \theta_{2}}\right)}\right|^{2}
\end{aligned}
$$

, where $\theta_{2}$ is the angle between the forward transition radiation wave vector and the beam axis, $\beta$ is the normalized electron velocity, e is the electron charge, and $\omega$ is the frequency of the 
radiation. To solve for the radiation emitted backwards into medium 1, we simply switch subscripts $1 \leftrightarrow 2$ and substitute $\beta \rightarrow-\beta$.

We can simplify the situation somewhat by noting that, in the case of an electron beam diagnostic, we commonly find that medium 1 is vacuum and $\varepsilon_{1}=1$. We are also more interested in the radiation directed forward from the material into vacuum than the radiation directed from vacuum into the material. We can rewrite eq. 3.1 as

$$
\frac{d^{2} I}{d \omega d \Omega}=\frac{e^{2} \beta^{2}}{\pi^{2} c} \frac{\sin ^{2} \theta \cos ^{2} \theta}{\left(1-\beta^{2} \cos ^{2} \theta\right)^{2}} \times\left|\frac{(\varepsilon-1)\left(1-\beta^{2}-\beta \sqrt{\varepsilon-\sin ^{2} \theta}\right)}{\left(\varepsilon \cos \theta+\sqrt{\varepsilon-\sin ^{2} \theta}\right)\left(1-\beta \sqrt{\varepsilon-\sin ^{2} \theta}\right.}\right|^{2}
$$

We can further simplify the situation by noting that, for relativistic electron beams, $\beta \rightarrow 1$. We will also typically be dealing with angles where $\theta \ll 1$, so we can make the small angle approximations $\sin ^{2} \theta \approx 0$ and $\cos \theta \approx 1$. We can also make the substitution $X=\beta \cos \theta$ (note that $X \approx 1$ ). If we make the approximation

$$
\frac{X^{2}}{\left(1-X^{2}\right)^{2}}=\frac{1}{\left(1+\frac{1}{X}\right)^{2}(1-X)^{2}} \approx \frac{1}{4(1-X)^{2}}
$$

and further note that $\beta \approx 1-\frac{1}{2 \gamma^{2}}$, we can rewrite eq. 3.2 as

$$
\frac{d^{2} I}{d \omega d \Omega}=\frac{e^{2}}{4 \pi^{2} c} \frac{\sin ^{2} \theta}{(1-\beta \cos \theta)^{2}} \approx \frac{e^{2}}{\pi^{2} c} \frac{\theta^{2}}{\left(\theta^{2}+\frac{1}{\gamma^{2}}\right)^{2}}
$$

, where we have also made use of the small angle approximations.

To solve for the radiation emitted backwards into the vacuum when the particle crosses from the vacuum into the medium, we can simply change $\beta$ to $-\beta$ in eq. 2.36 and apply the same simplifications as above to obtain

$$
\frac{d^{2} I}{d \omega d \Omega}=\frac{e^{2}}{\pi^{2} c} \cdot\left|\frac{\sqrt{\varepsilon}-1}{\sqrt{\varepsilon}+1}\right|^{2} \cdot \frac{\theta^{2}}{\left(\theta^{2}+\frac{1}{\gamma^{2}}\right)^{2}}
$$

This looks suspiciously like a Fresnel reflection term, which makes good physical sense - the waves that are emitted backward into the vacuum are those that cannot propagate in medium 2 and are thus reflected from the boundary [18]. Solving for the spectral intensity and plotting the results demonstrates that the radiation is strongly peaked at angle $1 / \gamma$ and is null at zero: 


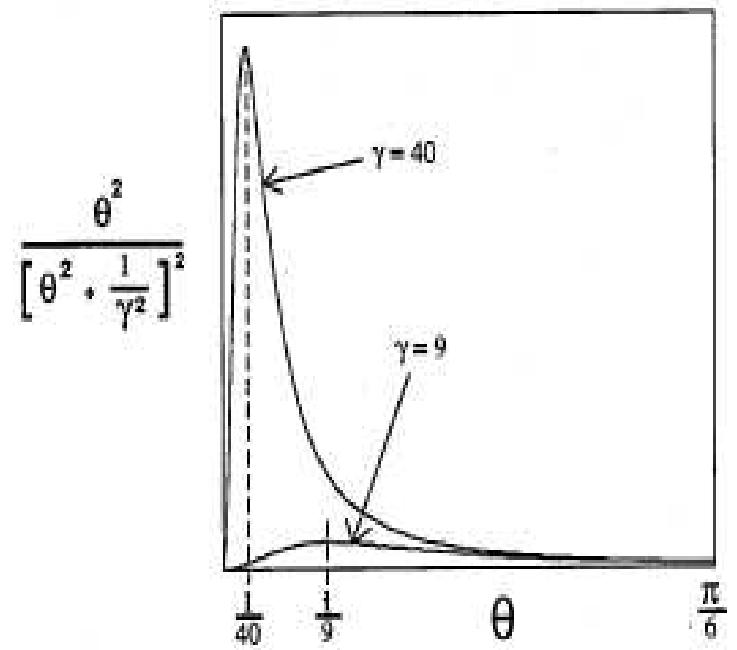

Figure 3.1. Angular dependence of the spectral intensity per unit solid angle at $4.5 \mathrm{MeV}$ and $20 \mathrm{MeV}$. From B.

Gitter [18].

. So far, we have considered only the case of particles crossing a foil at normal incidence. Unfortunately, this arrangement is not particularly useful from a beam diagnostic standpoint as we would need to place our detector directly in the beamline. A much more useful case arises if we incline our foil at a non-zero angle, in which case the backward radiation is emitted in the direction of specular reflection from the foil surface - if the foil is oriented at 45 degrees to the beam axis, the TR is emitted perpendicular to the beam where it can be easily imaged by a detector outside the beam pipe. Although Pafomov has developed the general formulae for oblique incidence [12], the equations are fairly unwieldy. Fortunately, for the case of optical frequencies in metals, we can apply image charge theory to the case of a perfectly conducting metallic foil. For each particle crossing the foil, we imagine that an antiparticle approaches from a trajectory along the direction of specular reflection from the foil. We can then consider transition radiation to be the radiation emitted from the pair annihilation process of these particles interacting at the foil, with the following expression for spectral intensity:

$$
\frac{d^{2} I}{d \omega d \Omega}=\frac{1}{4 \pi^{2} c}\left|\frac{-e \sin \theta}{1-\beta \cos \theta}+\frac{e \sin \theta^{\prime}}{1-\beta \cos \theta^{\prime}}\right|^{2}
$$

, where $\theta^{\prime}$ is the angle with respect to the image charge velocity, as shown below.

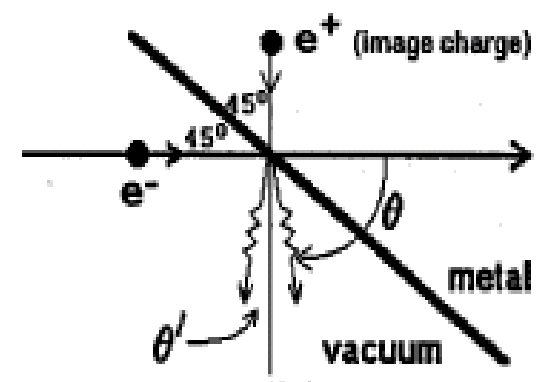

Figure 3.2. Image charge representation of back-emitted transition radiation. From B. Gitter [18]. 


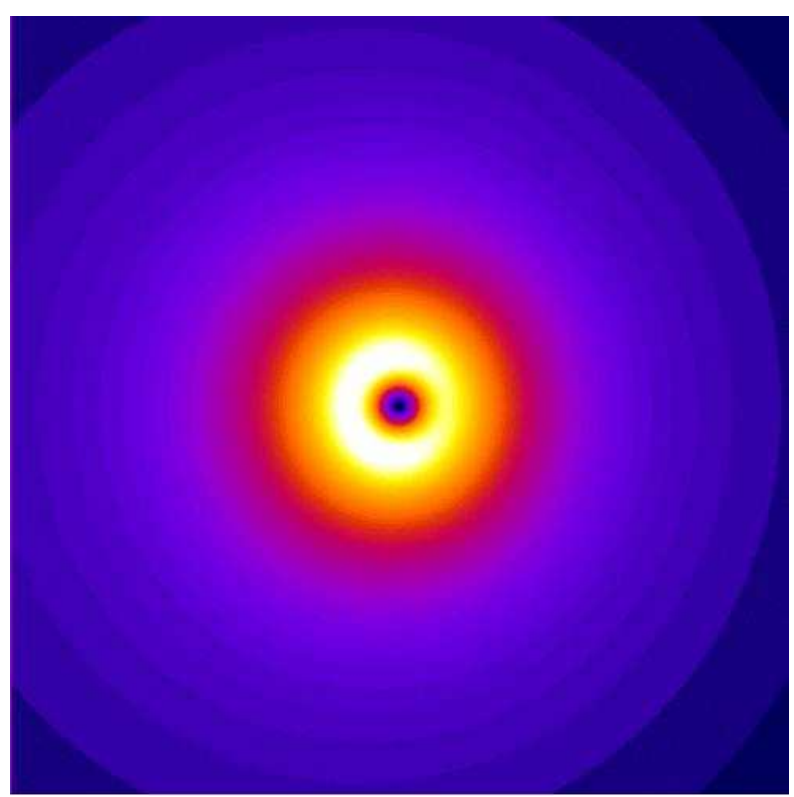

Figure 3.3. Theoretically predicted OTR angular distribution from a single electron. White is highest intensity, dark blue is lowest. From Carl Ekdahl's OTR Explorer software.

In Figure 3.3, we can clearly see the characteristic hollow-cone pattern with a well-defined peak at angle $1 / \gamma$ and a null at the center of the pattern.

In order to further analyze the pattern of the OTR light, we need to define the two different polarization directions, as shown below in Fig. 3.4. Lineouts of the OTR pattern in the parallel and perpendicular direction are shown in Fig. 3.5.

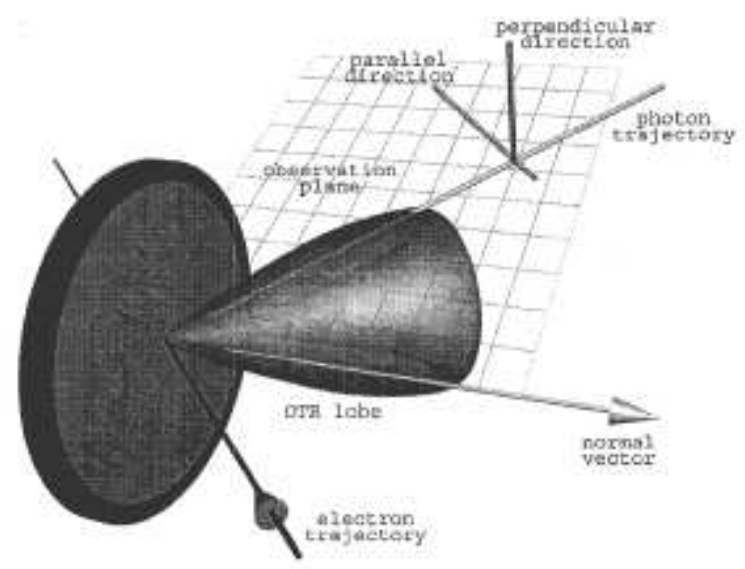

Figure 3.4. Definition of polarization directions. From C. Vermare and D. Moir [17]. 

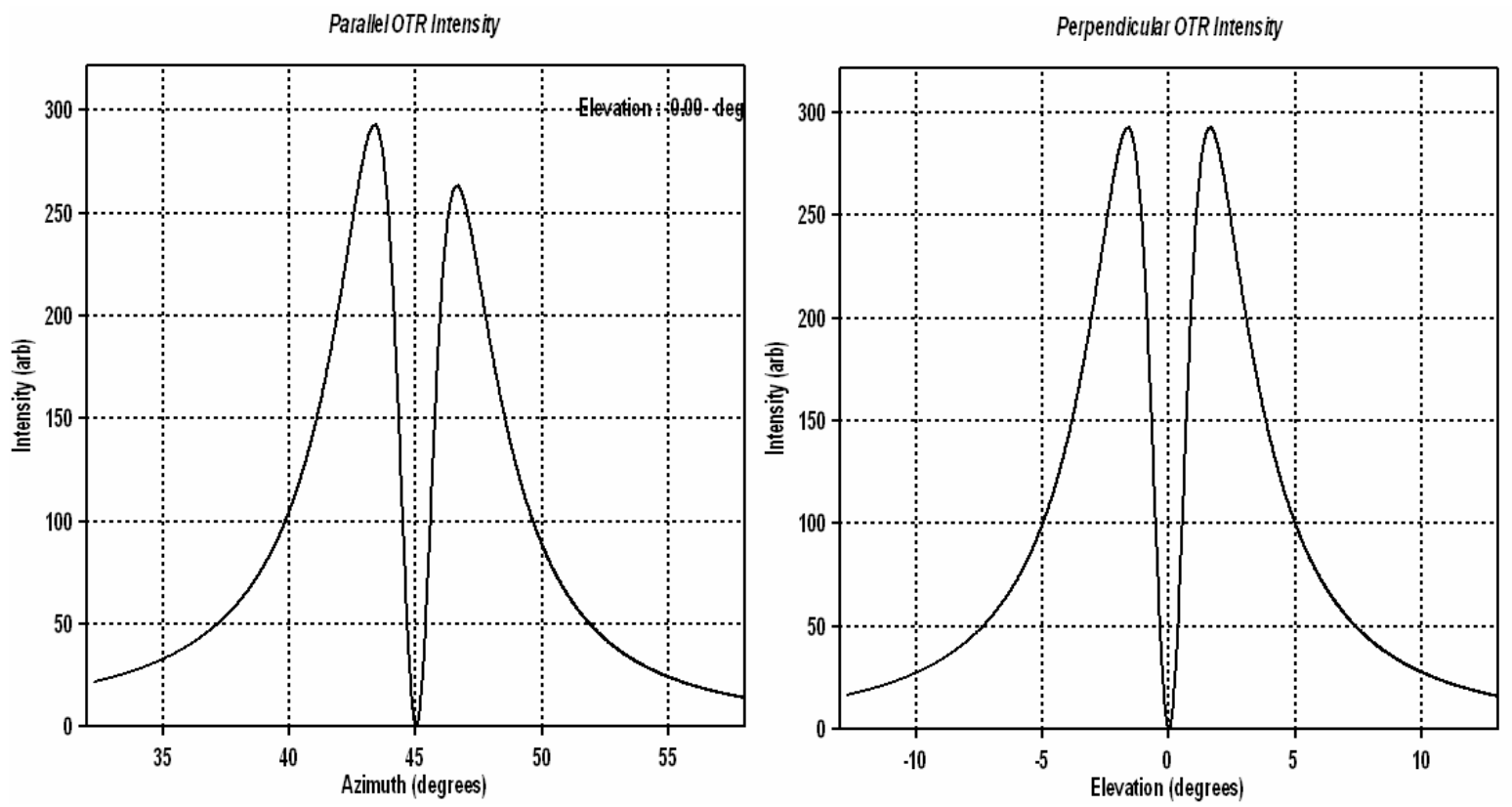

Figure 3.5. Lineouts of the OTR angular pattern in the parallel and perpendicular directions for a single electron at 17.5 MeV. From Carl Ekdahl's OTR Explorer software.

Clearly the OTR pattern is symmetric in the perpendicular direction but not in the parallel direction. From Eq. 3.6, we can see that the spectral intensity depends on the angle $\theta$; since the target foil is inclined relative to the beam axis in the horizontal (parallel) direction, $\theta$ varies between the two lobes in the parallel direction but not in the perpendicular direction. Returning to the Fresnel reflection term analogy, we recall that the reflection term is dependent on angle relative to the surface; qualitatively, we would expect that light at grazing incidence is reflected more strongly than light at normal incidence and this is, in fact, what we observe.

So far, we have analyzed the OTR light from a single electron crossing from vacuum into a thin metallic foil. Our real interest, of course, is in analyzing an electron beam, which is an ensemble of many individual particles. Thus, the OTR light produced by an electron beam is the superposition of many single-electron patterns. The center of the angular pattern is defined by the direction of specular reflection from the foil, which is determined by the incident angle of the electron. For a beam with nonzero emittance, each particle has a slightly different component of transverse momentum, which leads to a slightly different angle of incidence. Because of this, the single-electron OTR patterns from each particle are slightly offset from each other, leading to an overall blurring of the pattern. The degree of blurring is directly related to the angular spread of the beam and, therefore, the emittance. By utilizing a Monte Carlo simulation of an electron beam with a given divergence, we can produce the OTR angular pattern, as shown below. 


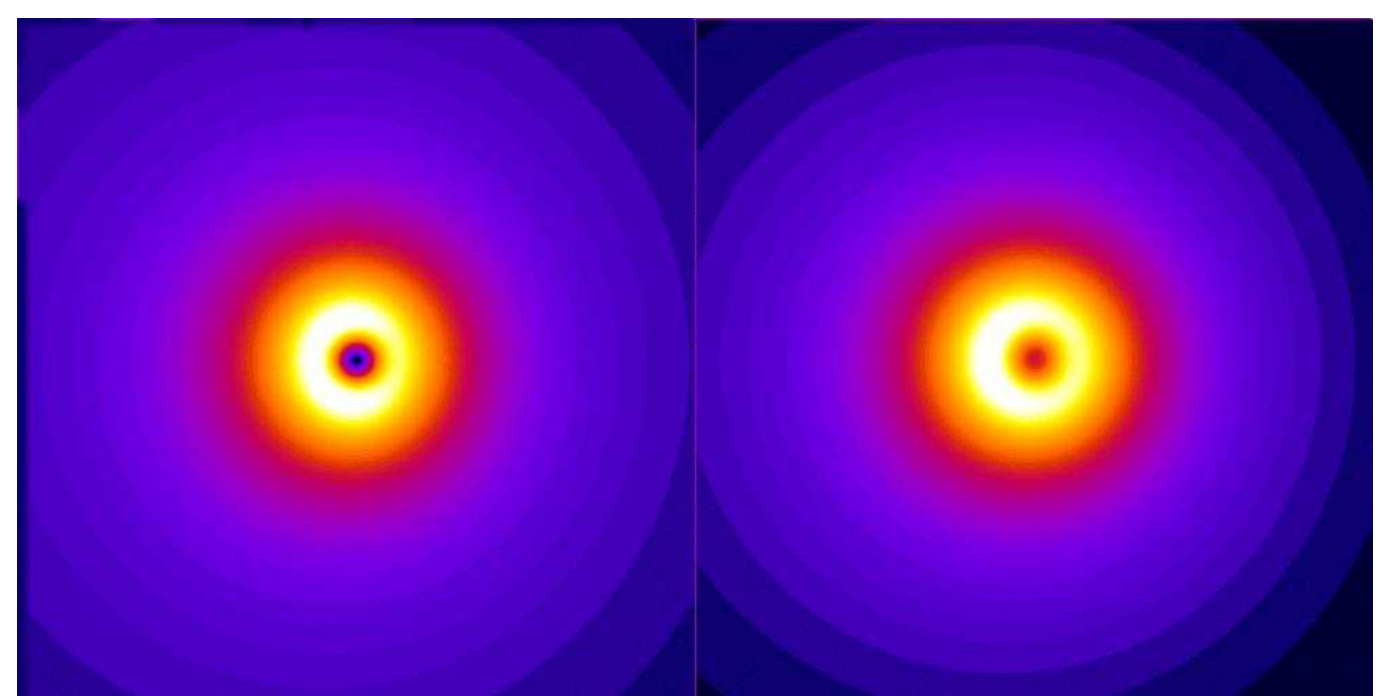

Figure 3.6. Comparison of OTR angular pattern from single electron at $17.5 \mathrm{MeV}$ (left) with beam at $17.5 \mathrm{MeV}$ and 15 mrad RMS divergence (right). White is highest intensity, dark blue is lowest. From Carl Ekdahl's OTR Explorer software.



Figure 3.7. Vertical lineout of OTR angular pattern for a single electron (red curve) and a beam with 15 mrad RMS divergence (blue curve). From G. LeSage [7].

Clearly, the most striking difference in the two patterns shown in Fig. 3.7. is the so-called valleyto-peak ratio, the difference in intensity between the peaks and the center minimum. This ratio is useful as an initial estimate of the divergence; an exact measure of the divergence can be obtained by fitting a theoretically prescribed curve for a given divergence to an empirical data set.

At this point, it is reasonable to question what effect the particular beam distribution will have on the observed OTR pattern. In the development of his OTR Explorer software, Carl Ekdahl (Los Alamos National Laboratory) has extensively investigated this issue and concluded that the details of the beam distribution have an insignificant effect on the OTR angular pattern; thus, it is possible to make an accurate emittance measurement without a priori knowledge of the particular distribution. Figure 3.8 below demonstrates that as the number of elements in the 
Monte Carlo simulation are increased, the valley-to-peak ratio converges to a single value for all beam distributions.

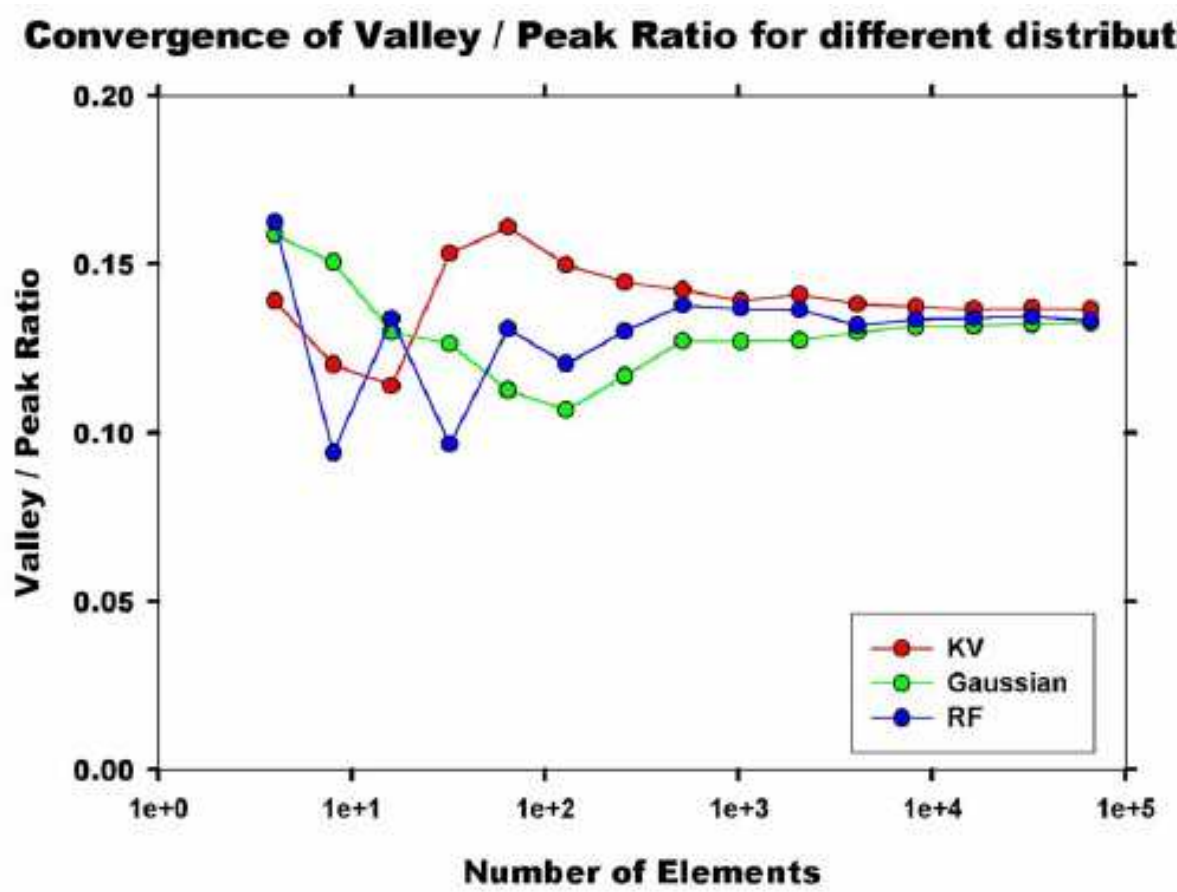

Figure 3.8. Comparison of valley-to-peak ratio for different beam distributions. Beam parameters are $20 \mathrm{MeV}, 1400$ mm-mrad normalized emittance, $5 \mathrm{~mm}$ RMS spot size. Red curve is Kapchinski-Vladamirski (uniform) distribution, green curve is Gaussian, blue curve is Rule-Fiorito distribution (Gaussian in angle, uniform in position). From C.

Ekdahl [23].

We may also question how the OTR angular pattern varies with wavelength. Simulation confirms that the pattern is essentially invariant across the optical spectrum, as shown below in Figure 3.9. Thus, there is no need to include any single-wavelength filters in the optical collection system.

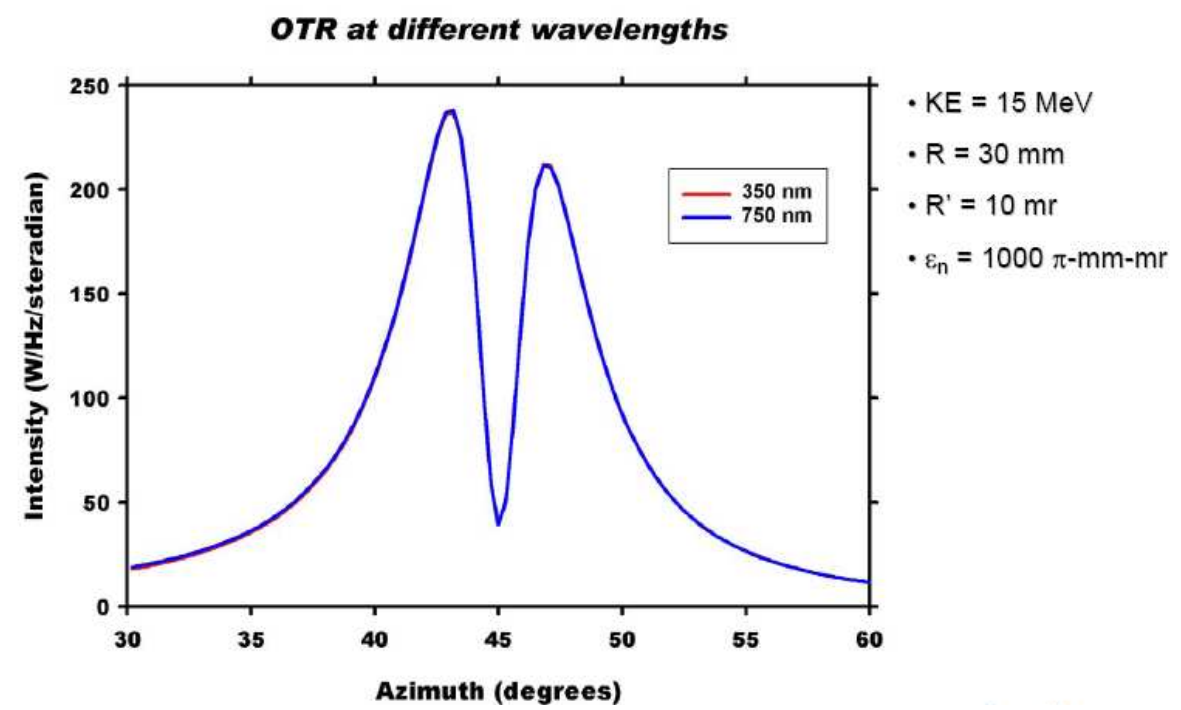

Figure 3.9. OTR angular pattern at different wavelengths. From C. Ekdahl [23]. 
The OTR angular pattern is also shown to be relatively invariant for any reasonable value of energy spread. Figure 3.10 below illustrates the pattern for values ranging from 17.325 to 17.675 $\mathrm{MeV}$, corresponding to a peak-to-peak energy spread of $2 \%$ about the nominal 17.5 MeV FXR beam energy; given recent energy analyzer measurements that suggest a $1 \%$ peak-to-peak spread, this is a fairly conservative worst-case estimate.

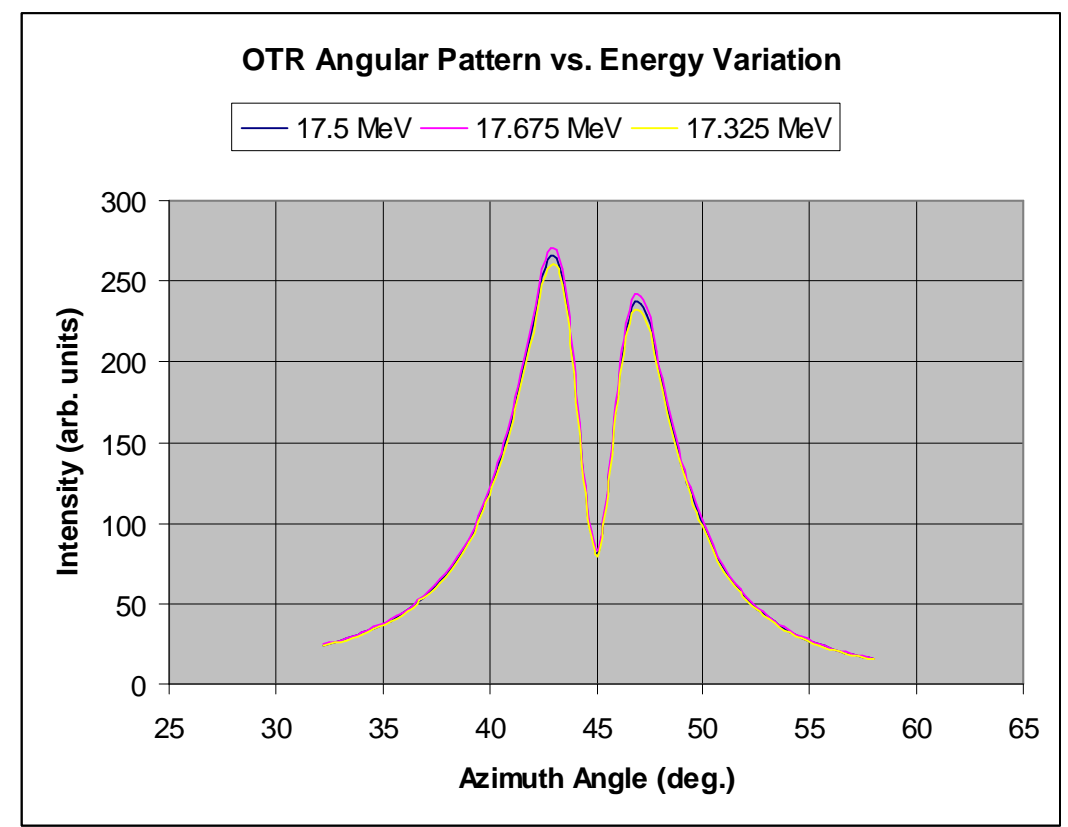

Figure 3.10. OTR angular pattern vs. energy variation. Beam divergence is $15 \mathrm{mrad}$ RMS.

\section{TARGET FOIL CONSIDERATIONS}

Due to the high current, long pulse length, and energy of the FXR beam, we must consider the effects of beam-induced heating on our target material. For the purposes of calculating the survivability of our foil, we consider the heating to be instantaneous, since the deposited power is orders of magnitude larger than the dissipation over the timescale of the beam interaction (70 ns). Energy is deposited through ionization and excitation of the target material according to the Bethe-Bloch formula,

$$
\left(\frac{d E}{d x}\right)=\frac{4 \pi N_{A} e^{4}}{m v^{2}} \frac{Z}{A}\left[\ln \left(\frac{2 m v^{2}}{I(1-\beta)^{2}}\right)-\beta^{2}\right]
$$

, where $\mathrm{m}$, e, and $\mathrm{v}$ are the mass, charge, and velocity of the electron, respectively, $\mathrm{N}_{\mathrm{A}}$ is Avogadro's number, $\mathrm{Z}$ and $\mathrm{A}$ are the atomic number and mass number of the atoms in the foil, I is an effective ionization potential $(=10 \mathrm{Z} \mathrm{eV}), \beta$ is the normalized velocity, and $\mathrm{x}$ is the path length in units of $\mathrm{g} / \mathrm{cm}^{2}, \sim 1.5 \mathrm{MeV} \mathrm{cm} / \mathrm{g}$ for most materials [18]. In order to find the energy loss per unit thickness of material, we simply need to multiply by the density. Note that the beam also loses energy due to bremsstrahlung and transition radiation as it crosses the foil, but this energy 
is not deposited in the foil - it leaves the foil in the form of EM radiation. Thus, the total energy deposited is given by

$$
\Delta E=N_{e}\left(\frac{d E}{d x}\right)_{i o n} \rho L
$$

, where $\mathrm{N}_{\mathrm{e}}$ is the number of electrons per shot $\left(\sim 1.25 \times 10^{15}\right.$ at FXR), $\rho$ is the density of the target material, and $\mathrm{L}$ is the thickness of the target foil. To calculate the heating of the material, we recognize that the energy deposited must equal the change in internal energy, $\Delta \mathrm{E}=\rho \mathrm{cV} \Delta \mathrm{T}$, where $\rho$ and $c$ are the density and heat capacity of the target material and $V$ is the volume of material intercepting the beam. Thus, we find that

$$
\Delta T=\frac{N_{e}\left(\frac{d E}{d x}\right)_{i o n}}{c A}
$$

, where $\mathrm{A}$ is the cross-sectional area of the foil intercepting the beam. Interestingly, this demonstrates that the instantaneous heating of the foil is independent of the foil thickness and density and depends only on the heat capacity. Given the characteristics of several candidate target materials, we have calculated the minimum spot size the material can tolerate, assuming a uniform distribution. The results are tabulated below.

\begin{tabular}{l|l|l|l|l|l} 
Material & $\begin{array}{l}\mathbf{c}(\mathbf{J} / \mathbf{g K}) @ \\
\mathbf{2 9 0} \mathbf{K}\end{array}$ & $\boldsymbol{\rho}\left(\mathbf{g} / \mathbf{c m}^{\mathbf{3}}\right)$ & $\begin{array}{l}\mathbf{d E} / \mathbf{d x} \\
(\mathbf{J} / \mathbf{c m})\end{array}$ & $\begin{array}{l}\text { Melting } \\
\text { point }(\mathbf{K})\end{array}$ & $\begin{array}{l}\text { Minimum } \\
\mathbf{x}_{\mathbf{r m s}}(\mathbf{m m})\end{array}$ \\
\hline Quartz & 0.739 & 2.64 & $6.34 \mathrm{E}-13$ & 1700 & 2.46 \\
\hline Mylar & 1.17 & 1.39 & $3.35 \mathrm{E}-13$ & 527 & 3.49 \\
\hline Kapton & 0.747 & 1.42 & $3.41 \mathrm{E}-13$ & 793 & 3.01 \\
\hline Aluminum & 0.897 & 2.70 & $6.49 \mathrm{E}-13$ & 934 & 3.35
\end{tabular}

Table 4.1. Thermal properties and minimum tolerable spot size for various target materials. Note that Kapton does not melt, but decomposes at $793 \mathrm{~K}$.

\section{EXPERIMENTAL SETUP AT FXR}

\section{Hardware}

Our diagnostic at FXR consists of three basic components: the target foil, the optical collection system, and the camera. For our target, we chose a quartz foil with 0.015 inch thickness. Although Kapton, Mylar, and other plastic materials are available in thinner sheets, quartz has the advantage of hardness and surface flatness, compared to the tendency of some thin films to wrinkle and bubble; quartz is also the most robust choice in terms of thermal loading. The upstream-facing side of our foil was polished and coated with 1000 Angstroms of Aluminum using a high-precision vacuum deposition process in order to provide an optical quality surface for observing the OTR angular pattern. The back side of the foil was ground to a frosted texture 
(64 microinch finish) such that Cerenkov light generated in the bulk of the foil scatters from the frosted surface, allowing easy imaging of the beam spatial profile. Quartz also afforded us the possibility of simultaneously observing the OTR angular distribution and the beam spatial profile for single-shot emittance measurement, as shown below:



Figure 5.1. Simultaneous observation of beam divergence and spatial profile. From G.P. LeSage [7].

In this arrangement, the OTR angular distribution is imaged from the aluminized side of the foil, while scattered Cerenkov light is imaged from the frosted quartz side to obtain the spot size, allowing true single-shot measurement of both the angular divergence and the spatial profile of the beam and, hence, the emittance. Unfortunately, the lack of a second camera during our experimental runs prevented us from utilizing this feature, and angular spread and spot size were imaged separately by exchanging lens packages. The capability for single-shot measurements, however, can be easily implemented in the future.

The foil was mounted in a 4.375 " diameter black Delrin holder, which was then attached to a manually operated linear inserter in the diagnostic cross at the downstream end of the FXR accelerator. In order to minimize scattered background light, the interior of the cross was anodized black. Proper orientation of the foil was verified by launching a HeNe alignment laser down the bore of the accelerator from an in-vacuum mirror near the injector and adjusting the orientation of the foil such that the laser was reflected out of the diagnostic cross at 90 degrees to the beam axis.

In order to collect a suitable portion of the OTR angular pattern, our lens system was designed using the OSLO computer code to collect a full angle of at least $8 / \gamma$, or about 13 degrees. OSLO simulation also verified that the full range of light would be collected from every point in the finite spatial profile of the beam so as to not add a spatial convolution to the resulting data; care was also taken to minimize aberrations in the focusing system. Early OTR-based emittance 
measurements at FXR utilized a system of 8" diameter collection lenses mounted to an optical table breadboard outside the diagnostic cross [7]. While this setup was effective, it was prone to accidental misalignment and was difficult to focus. In order to ease the installation and setup of a production OTR diagnostic, we have utilized a self-contained lens package consisting of a black Delrin cylinder housing two 4" diameter positive achromat lenses ( $\mathrm{f}=500 \mathrm{~mm}$ ) which project onto a frosted glass diffuser screen at the rear of the cylinder. The focal length of the package is adjustable by sliding the diffuser screen in and out of the main tube and can be locked by tightening two set screws. For observation of the OTR angular pattern, the diffuser screen is located at the focal length of the lens package such that the system is focused at infinity; in this configuration, all rays of a given direction map to a single point regardless of their spatial point of origin. The entire assembly is held in place by an aluminum flange mounted directly to the diagnostic cross such that the collection lenses are flush against the viewport on the side of the cross and are as close as possible to the OTR target foil - this allows us to use smaller, lighter, less expensive lenses while still collecting the requisite amount of light. On the raytrace simulation shown below, the rays originate from different points on a $10 \mathrm{~mm}$ spot and at different angles - the green rays are axial, while the red and blue rays are emitted at $+/-4 / \gamma$. The mapping of incident angle to detector position is clearly demonstrated.

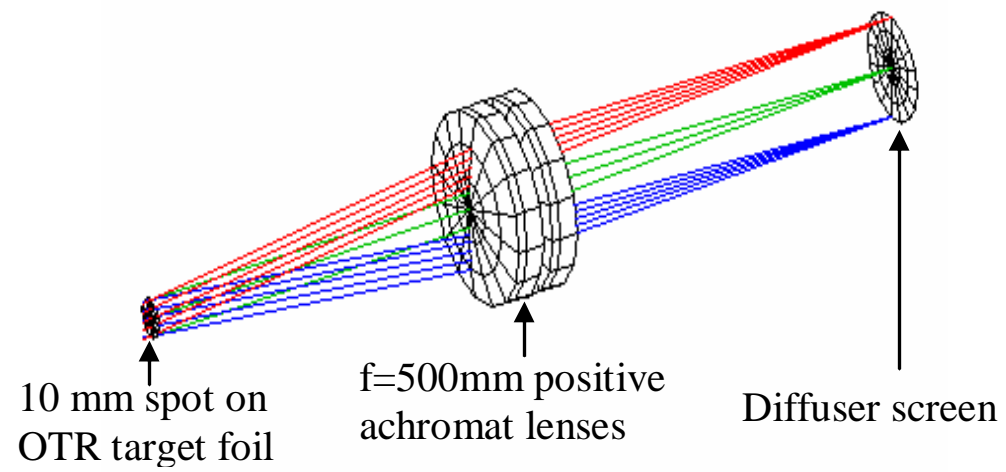

Figure 5.2. Raytrace simulation of angular data collection optics using OSLO.

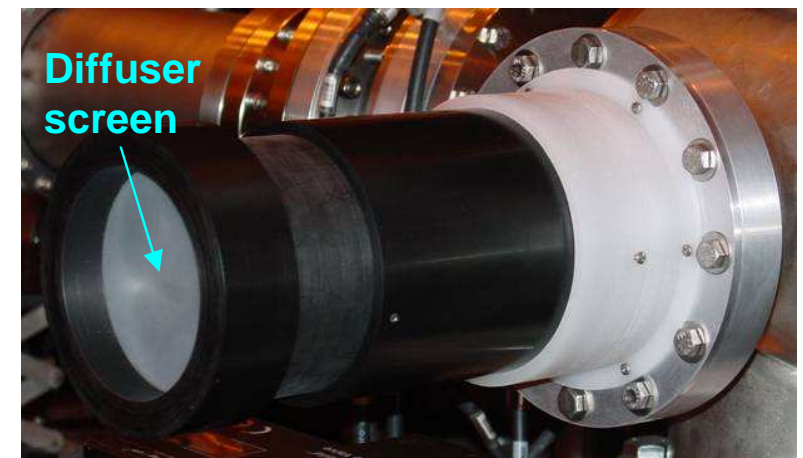

Figure 5.3. OTR lens package

For connection to our camera system, a conventional $35 \mathrm{~mm}$ camera lens was mounted in a Delrin holder and an adapter flange was manufactured to mount the camera lens to the OTR package at the proper focal length such that the entire system simply slides together with no adjustment required. Angular calibration of the system was achieved on the tabletop by 
launching a low-power laser into the collection lens at a known angle and mapping input angle vs. location on the detector, yielding a result of 39.75 pixels / degree and confirming a total field of view of 25.76 degrees or $15.8 / \gamma$, comfortably beyond the minimum requirement. Although this should provide more than acceptable resolution, it may be desirable to reduce this field of view in the future in order to enhance the resolution of the angular pattern.

Our camera of choice was a Princeton Instruments PI-SCX with <5ns gating, >1000:1 contrast ratio (with thermoelectric cooling), 1024x1024 pixel resolution, and 16 bit dynamic resolution. The camera was enclosed in a metal box surrounded by lead for x-ray and EMI shielding and was located approximately two meters to the side of the beamline so as to be clear of the direct bremsstrahlung path of the linac. The camera was combined with a high-speed Gated Optical Intensifier to allow exposure times down to $1 / 2 \mathrm{~ns}$. The GOI comprises a photocathode, a micro channel plate (MCP), and a high-voltage electrical pulsing system. The GOI has an equivalent 15 bit dynamic range based on a CCD noise floor of approximately 600 counts and a nonlinear response above approximately 20000 counts with the GOI sensitivity set to maximum and the CCD analog gain set to medium. The CCD was linked to the optical collection system through a fiber bundle made of $400 \times 40010 \mu \mathrm{m}$ diameter fibers [7].

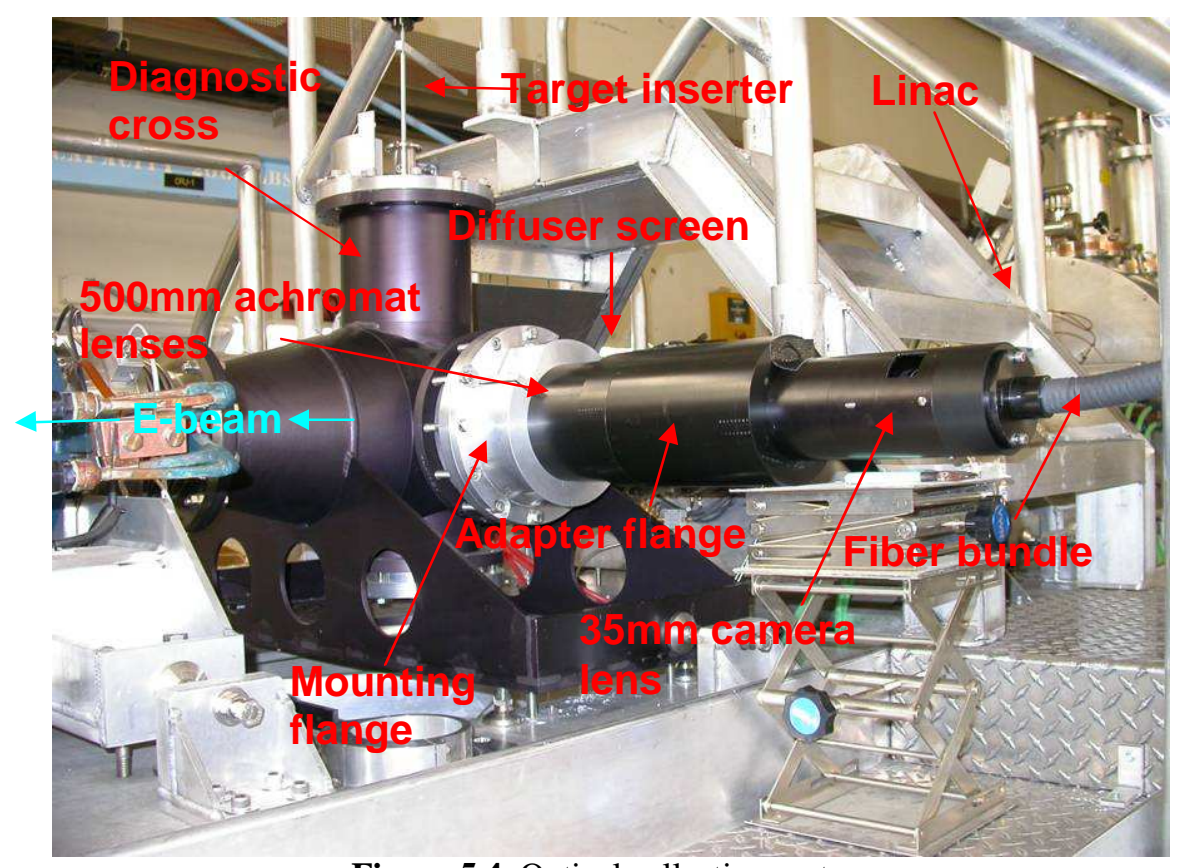

Figure 5.4. Optical collection system

\section{Software}

Initial collection of raw data was accomplished through the Roper Scientific WinView32 proprietary software; this program provides not only image capture capabilities but provides access to all of the camera hardware settings, including detector temperature, analog gain, exposure time, etc. Images are stored in the proprietary SPE format, which provides 16-bit $1024 \times 1024$ resolution and also records the camera settings for each image. A screen shot of the program with raw spot size and OTR angular images is shown below. 


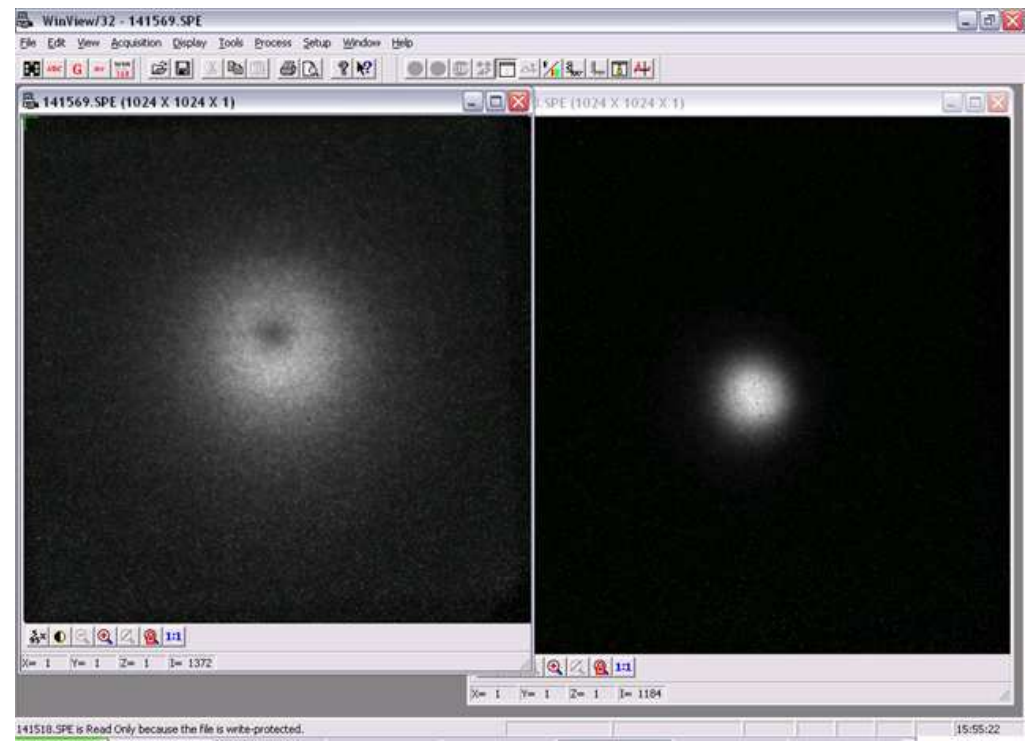

Figure 5.5. Raw data images in WinView32 software.

RMS spot size measurement was accomplished through a LabVIEW VI provided by Greg LeSage and Scott Anderson. Given a raw image in TIFF format, the VI will perform a Gaussian fit to the data and calculate both the Gaussian sigma and the RMS size of the beam, as shown below.

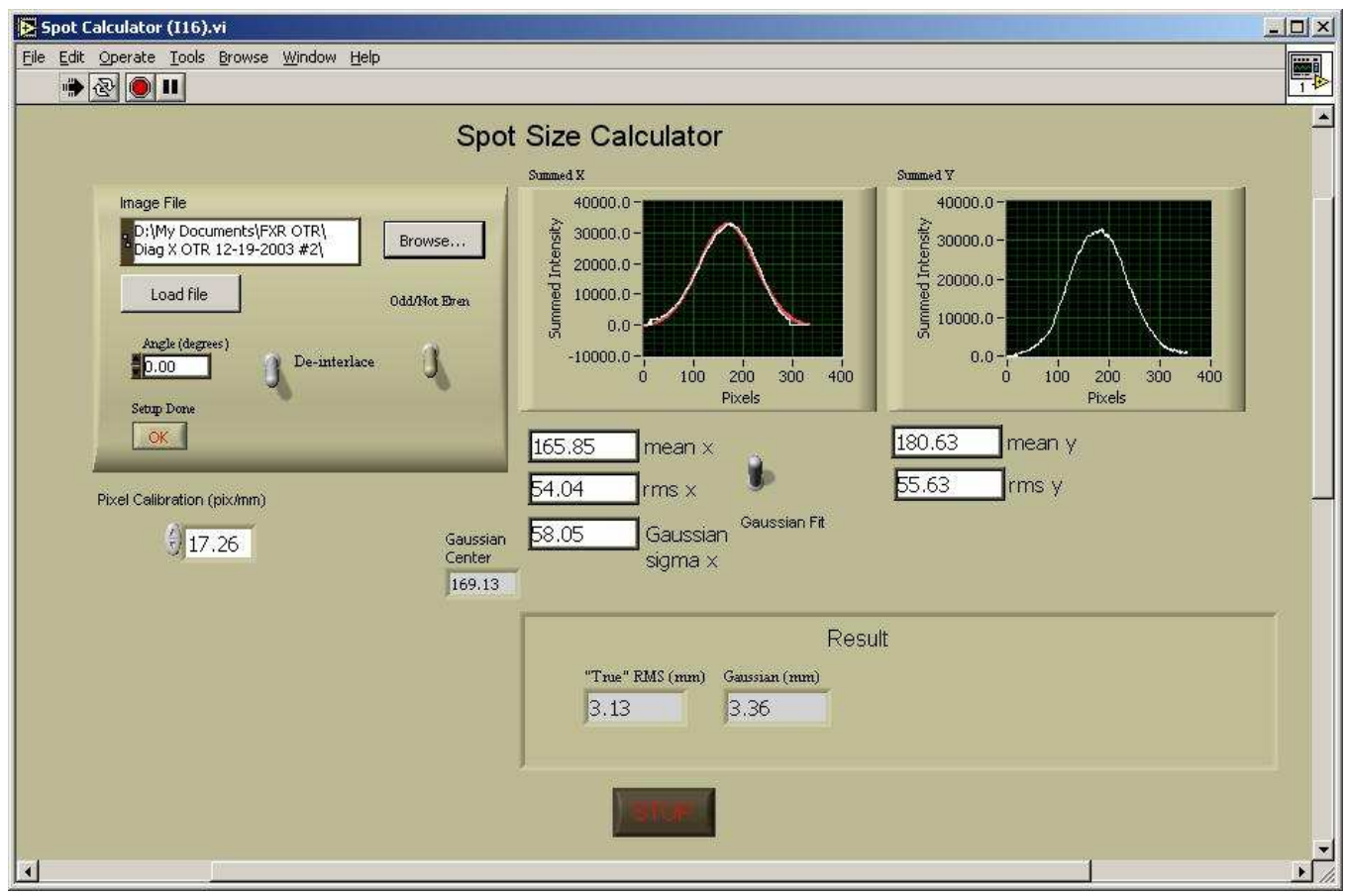

Figure 5.6. Spot size calculation software from Greg LeSage and Scott Anderson.

Initial processing of the OTR angular data imagery was accomplished through another customdesigned LabVIEW VI. The software first performs a low-pass filter on the entire image to remove radiation speckle noise - each pixel is compared to its neighboring pixels and, if the 
difference exceeds a given percentage, the offending pixel is replaced with the average value of its neighbors. The percentage threshold and filter size are adjustable on the front panel of the VI and were optimized to provide the most effective filtering with the least impact on the overall image pattern. Next, the software locates the center of the OTR angular pattern and performs a lineout in both the horizontal and vertical directions. A median filter is then applied to the lineout data to smooth out irregularities; again, the filter settings are adjustable via the front panel of the VI and were optimized to provide the most effective filtering with the least impact on the overall pattern. Finally, given an angular calibration, the VI takes the horizontal lineout data, converts it to intensity vs. azimuth angle from the surface of the foil, and stores the data as a two-column text file; a rough estimate of the beam energy (calculated from the spacing of the peaks) and the valley-to-peak ratio is also provided.

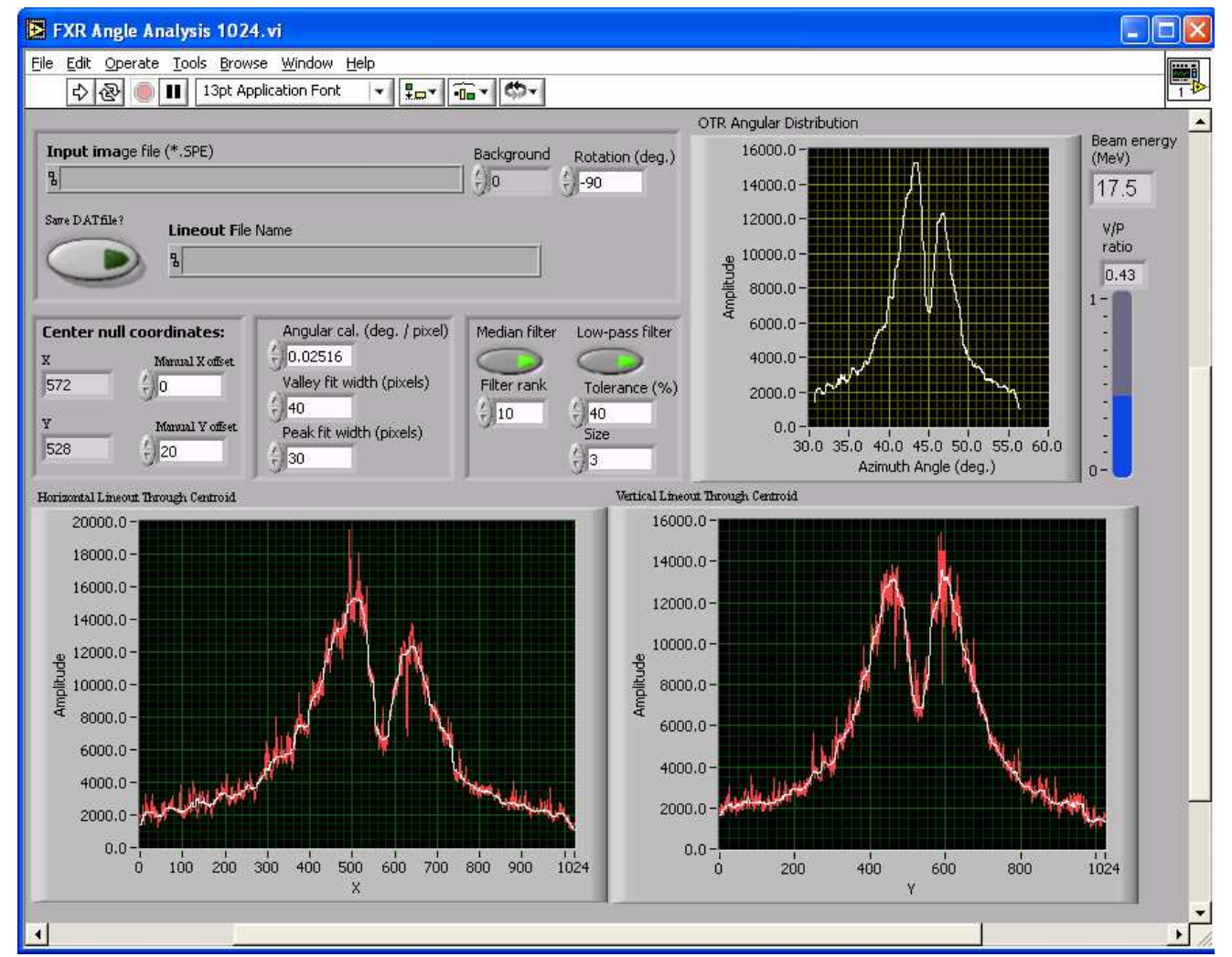

Figure 5.7. Image processing LabVIEW VI for OTR angular data. In the horizontal and vertical lineouts of the OTR angular pattern at the bottom of the image, the red curve is raw data, while the white curve is the data after filtering. A plot of intensity vs. azimuth angle is shown in the upper right

The final step in emittance calculation utilizes the OTR Explorer software provided by Carl Ekdahl of Los Alamos National Laboratory. The software takes the horizontal lineout data and the RMS spot size measurement as input and, using the valley-to-peak ratio of the OTR angular distribution as an initial guess, performs a maximum-likelihood two parameter fit to yield the normalized emittance. The basis of the code is the single-electron OTR theory developed by V.E. Pafomov [12]. Extensive testing of the code has been performed by Carl Ekdahl at the Los Alamos DARHT facility to ensure internal consistency as well as consistency with other OTR codes and other measurement techniques [23]. As discussed in the theory section, the OTR angular pattern is nearly independent of the exact details of the beam distribution, and so an 
accurate emittance measurement can be made without knowing the exact characteristics of the of the beam distribution at FXR; any reasonable value of energy spread also produces a negligible effect in the OTR pattern.



Figure 5.8. Carl Ekdahl's OTR Explorer software.

\section{DATA REDUCTION}

Our camera system was configured with the following parameters for data collection:

- $\quad$ CCD temperature $=-30 \mathrm{C}$

- $\mathrm{CCD}$ exposure time $=0.5 \mathrm{sec}$

- GOI exposure time $=1 \mathrm{~ns}$

The initial step before collecting and analyzing any actual data was to verify the correct timing of the camera trigger. With the spatial imaging lens package installed, we took three exposures: one at what we believed to be the center of the beam pulse, one $60 \mathrm{~ns}$ ahead of center, and one $60 \mathrm{~ns}$ after center in order to bracket the $70 \mathrm{~ns}$ beam pulse. As expected, we observed beam at the center of the pulse and no beam $60 \mathrm{~ns}$ off-center in either direction. After collecting additional spot size data, we analyzed the total counts on the CCD in a region around the beam profile in order to compare to the known current profile; since only 5 different temporal locations were investigated, a sixth-order polynomial was fit to the data points to yield a complete curve. The results are shown in the charts below. 


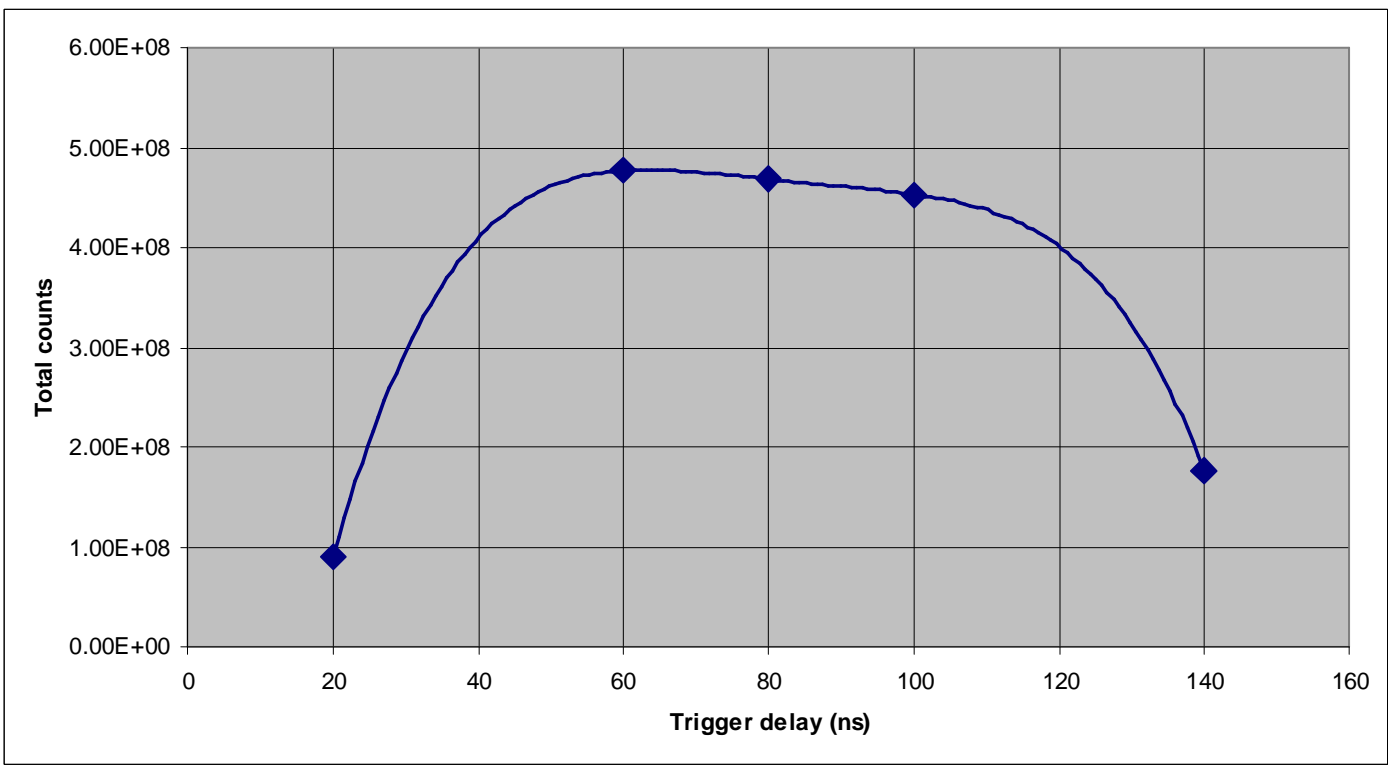

Figure 6.1. CCD counts vs. camera trigger delay

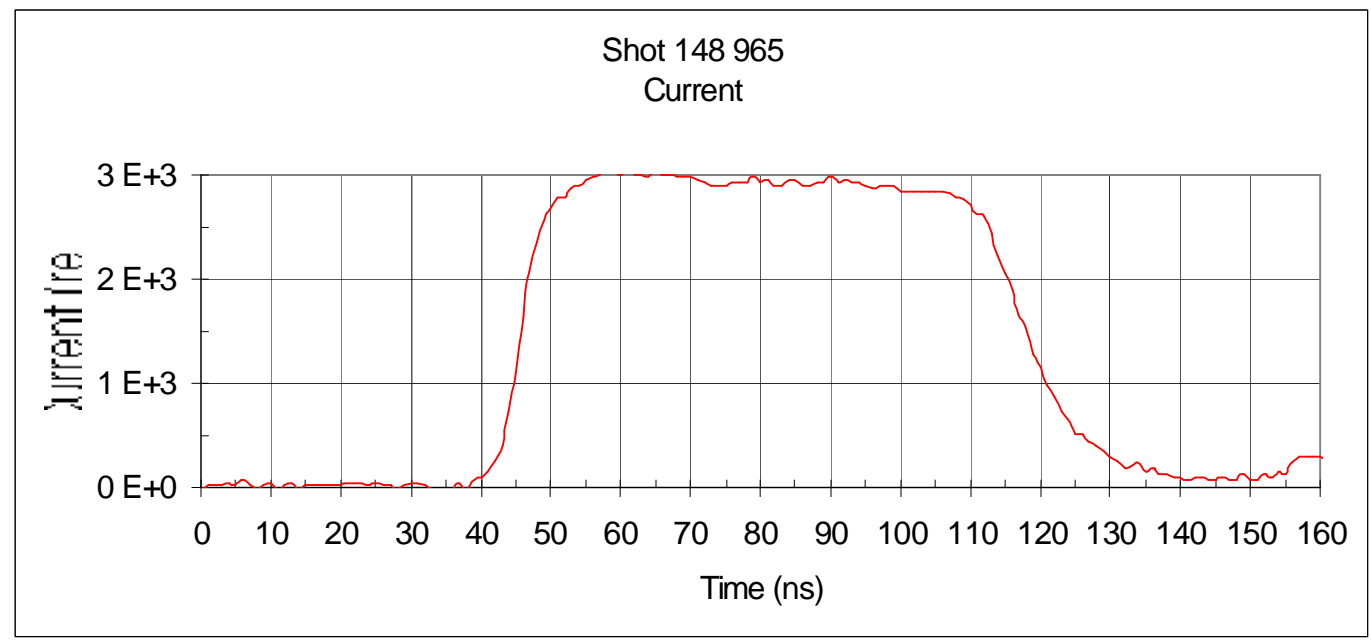

Figure 6.2. Beam pulse current measurement

Given the obvious similarity in the shape of the pulses shown in Figs. 6.1 and 6.2, we can conclude that our trigger timing is correct; although the width of the pulse is approximately 100 ns FWHM in Fig. 6.1, additional data points would likely yield a curve more similar to the $70 \mathrm{~ns}$ FWHM pulse shown in Fig. 6.2.

The next step was to adjust transport magnet settings to focus the beam to a waist at the OTR target foil, which was accomplished by adjusted the current in the DR1 and DR2 solenoids, which are the first transport magnets in the drift section immediately following the linac. In order to produce a relatively large spot size at the waist so as to minimize the chance of damage to the foil, we chose to focus the beam hard as far upstream as possible. DR1 was set at its maximum current of $425 \mathrm{~A}$ while DR2 was scanned through a range from 0 to $425 \mathrm{~A}$, and the RMS spot size was calculated at each point. The measurement was repeated at three separate temporal 
locations in the beam - once in the center of the $70 \mathrm{~ns}$ beam pulse, once at $20 \mathrm{~ns}$ ahead of center, and once at $20 \mathrm{~ns}$ behind center. The results are shown in the chart below.



Figure 6.3. RMS spot size as a function of DR2 current. The blue curve, with $80 \mathrm{~ns}$ camera trigger delay, represents the center of the beam pulse, the pink curve the head, and the yellow curve the tail.

Clearly, the focusing of the beam is distinctly different at different times within the beam pulse; this would seem to indicate a variation in energy and / or emittance between the head and tail of the beam. Although the waist appears to occur at slightly different focusing strengths depending on the temporal location within the pulse, we selected DR2 $=200 \mathrm{~A}$ as the optimal setting to achieve a waist at the OTR foil. The RMS spot size measurements at 60, 80, and $100 \mathrm{~ns}$ camera trigger delay were then utilized for the RMS emittance calculation; due to run time constraints, we were limited to one measurement at each temporal location.

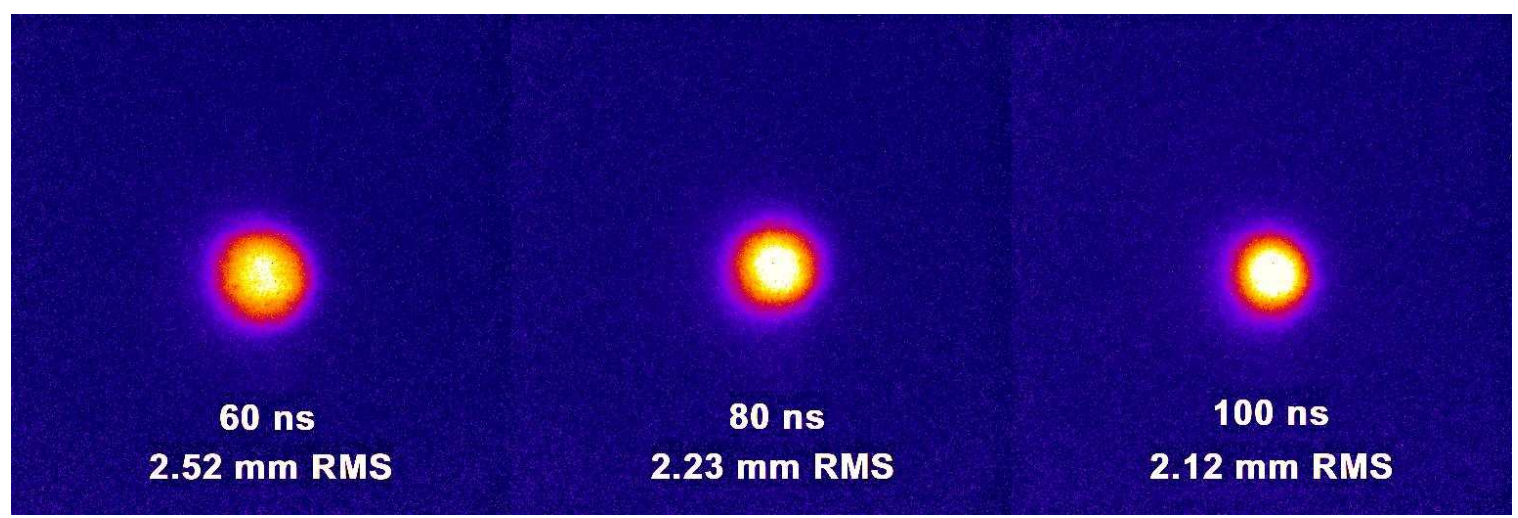

Figure 6.4. Beam profile images at 60,80 , and $100 \mathrm{~ns}$ camera trigger delay.

It is worth noting that a lineout of the spot size images demonstrates that the distribution is almost perfectly Gaussian, as shown below. This is to be expected from an emittance-dominated beam. 


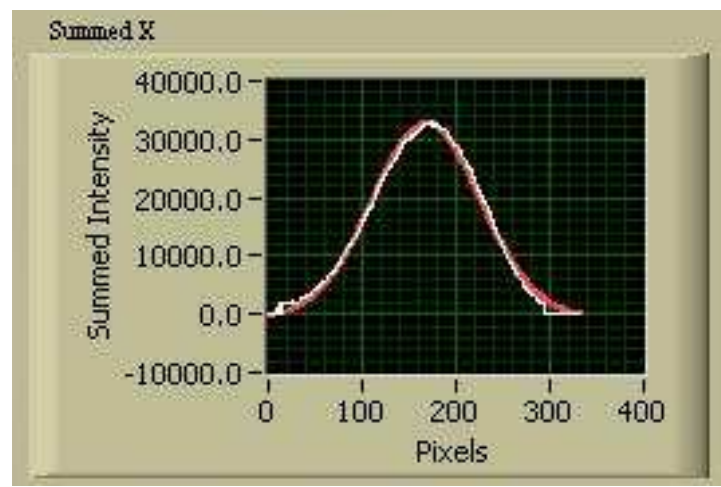

Figure 6.5. Spot size analysis showing experimental data from FXR (white curve) and best Gaussian fit (red curve).

After locating the beam waist, the angular lens package was installed and angular data collected at 60, 80, and $100 \mathrm{~ns}$ camera trigger delay. The raw data was processed in the LabVIEW VI described above and the resulting lineout data was analyzed in Carl Ekdahl's OTR Explorer software to yield the emittance and angular spread. The chi-squared value of the fit to each of the images shown below was approximately a factor of 3 lower than the sample data provided with the OTR Explorer software, indicating a good match between theory and measurement. Initial observation of the angular spacing between the peaks of the OTR pattern confirms a beam energy of $\sim 17.5 \mathrm{MeV}$.

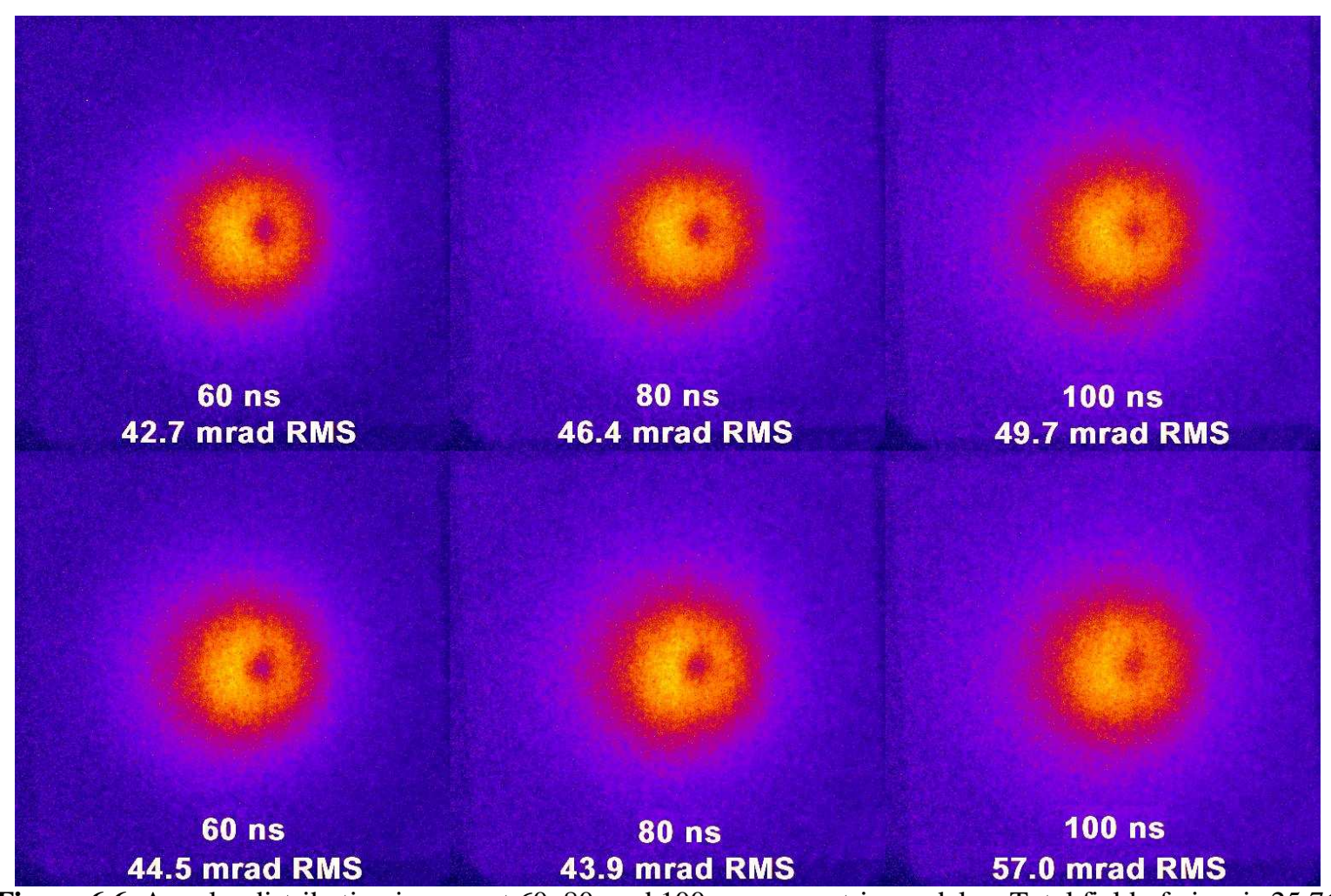

Figure 6.6. Angular distribution images at 60, 80, and $100 \mathrm{~ns}$ camera trigger delay. Total field of view is 25.76 degrees in both dimensions. 
The faint vertical line visible in each image is presumed to be caused by a darkening of one of the layers of the optical fiber bundle - during the manufacturing process, flat rows of fibers are fused together and stacked atop each other layer by layer, forming the overall two dimensional grid of the bundle; apparently, one layer was either originally somewhat opaque or is somehow more susceptible to radiation-induced darkening. Fortunately, the effect on the overall pattern is minimal.



Figure 6.7. Horizontal lineout of OTR angular distribution. Azimuth angle is measured from the surface of the target foil in the horizontal dimension.

The lineout data shown above is the final result after low-pass and median filtering. The spacing of the peaks is approximately 1.6 degrees or $28 \mathrm{mrad}$, which corresponds to a beam energy of $\sim 17.5 \mathrm{MeV}$. 


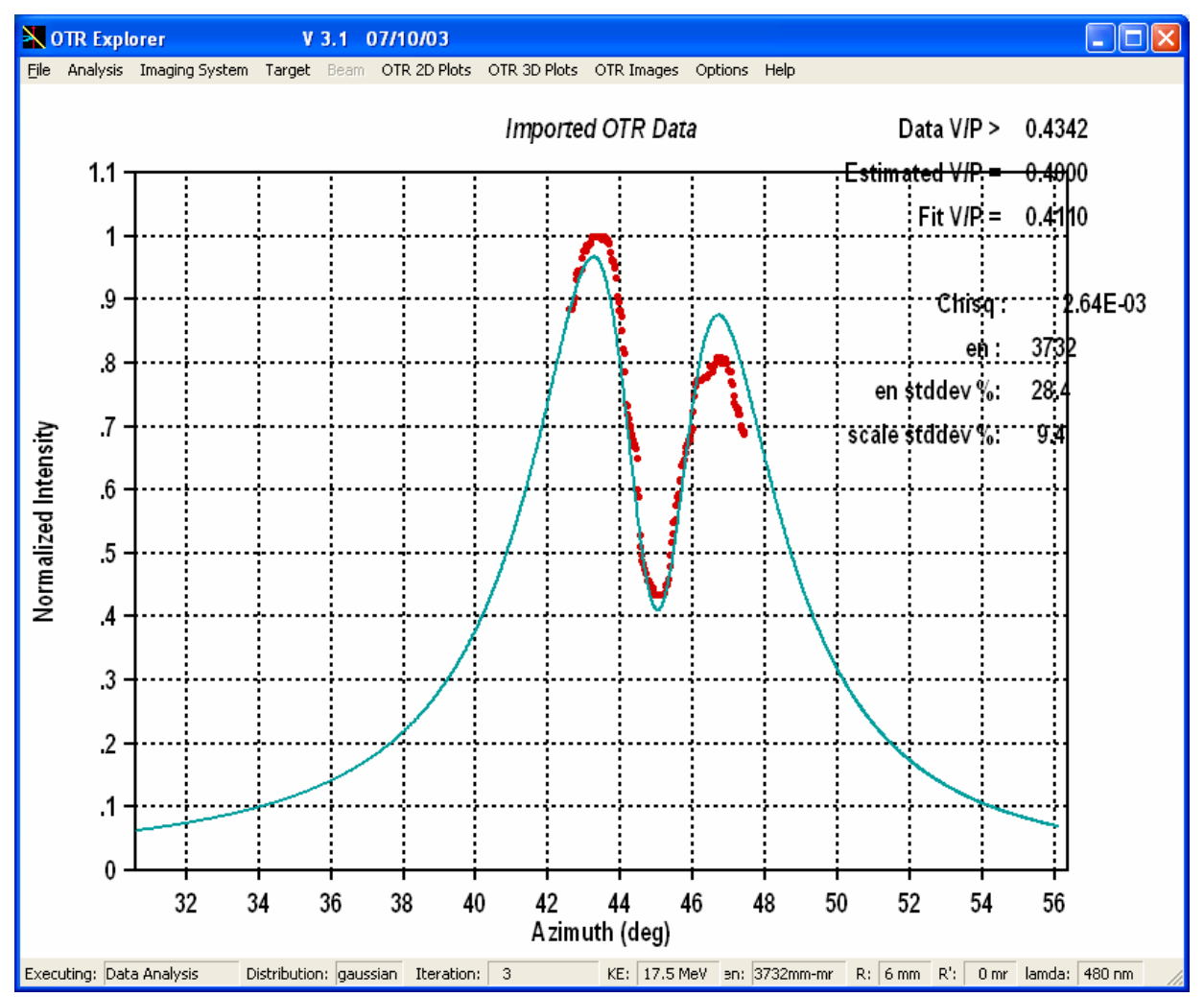

Figure 6.8. Least-squares fit in OTR Explorer software. Red curve is empirical data, blue curve is analytic fit.

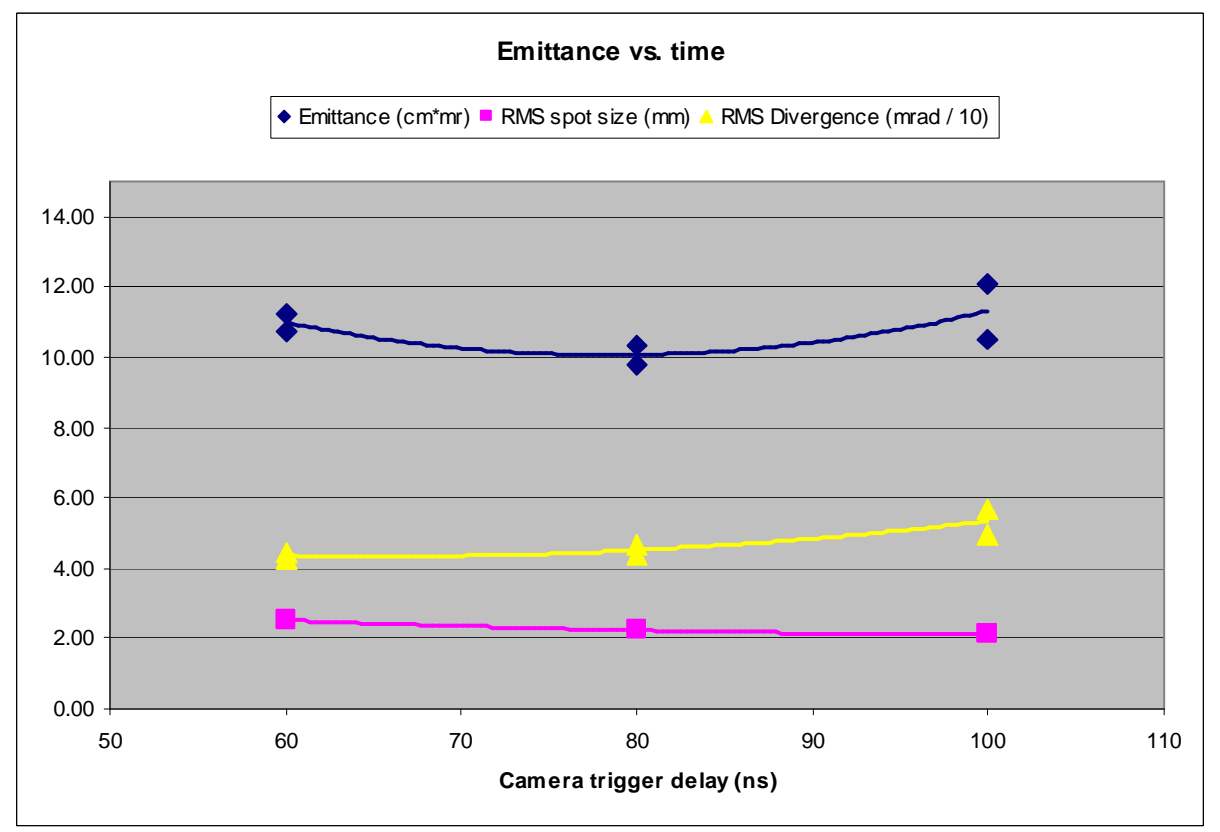

Figure 6.9. RMS spot size (pink curve), RMS divergence (yellow curve), and RMS emittance (blue curve) as a function of time. 


\section{CONCLUSIONS}

Optical transition radiation has been verified as a viable emittance measurement technique for the FXR beam - our empirical measurement of the OTR angular pattern matches theoretical predictions very well, and our measured emittance values are in good agreement with previous OTR measurements as well as previous magnet scan measurements, as shown in Table 7.1.

\begin{tabular}{|c|c|}
\hline Measurement Type & $\begin{array}{c}\text { Average Normalized RMS Emittance } \\
\text { (cm-mr) }\end{array}$ \\
\hline Magnet scan [6] & 400 \\
\hline OTR (G. LeSage [7]) & 370 \\
\hline OTR (J. Jacob et. al.) & 380 \\
\hline
\end{tabular}

Table 7.1. Summary of recent emittance measurements at FXR

As noted in the introduction and illustrated in Table 1.1, the measured emittance at FXR is both considerably higher than simulations predict and also much higher than measured values at other similar facilities. Given the inability of simulations to accurately model the physics of nonuniform cathode emission, which is the suspected root cause of the higher-than-expected emittance at the injector exit [24], a robust and accurate emittance measurement system is required to provide empirical feedback on the efficacy of new accelerator tunes and hardware improvements; we believe we have demonstrated that our OTR diagnostic fulfills this role. The next logical step for OTR measurement is to further investigate the temporal evolution of the emittance throughout the beam pulse, and also to begin empirical measurements of emittance vs. cathode gap, bucking coil field, injector solenoid fields, and other hardware settings that may have a significant impact on the emittance of the beam at the injector exit. In the future, the OTR diagnostic can be significantly improved by adding a second camera to facilitate the simultaneous collection of spatial and angular data, thereby allowing true single-shot emittance measurement.

\section{ACKNOWLEDGEMENTS}

The authors would like to thank Jan Zentler, Jim Dunlap, Ken Griffin, Keith Lewis, and the entire FXR staff for their continuing help and support. We would also like to thank Carl Ekdahl of Los Alamos National Laboratory for his OTR analysis software, Lynn Seppala for his help with optics design, Scott Anderson for contributions to spot size measurement software, and Donald Fleming and Nicholas Hash for their mechanical expertise. 


\section{REFERENCES}

[1] R. Scarpetti, J. Zentler, J. Boyd, G. Earley, R. Kerr, R. Kihara, K. Griffin, "Upgrades to the LLNL Flash X-Ray Induction Linear Accelerator (FXR)," $11^{\text {th }}$ IEEE International Pulsed Power Conference, Baltimore, MD, June 29-July 2, 1997

[2] G.P. Le Sage, S.G. Anderson, T.E. Cowan, J.K. Crane, T. Ditmire, J.B. Rosenzweig, "RF photoinjector development for a short-pulse, hard X-ray Thomson scattering source," AIP Conference Proceedings, (no 569), p.391-404, 2001

[3] S. Humphries, Charged Particle Beams, John Wiley and Sons, Inc., 1990

[4] J. Rosenzweig, G. Travish, "Design considerations for the UCLA PBPL slit-based phase space measurement system," March 2, 1994, Technical Note online at http://pbpl.physics.ucla.edu/Literature

[5] H. Wiedemann, Particle Accelerator Physics: Basic Principles and Linear Beam Dynamics, Springer-Verlag, New York, 1993.

[6] W.E. Nexsen, R.D. Scarpetti, J. Zentler, "Reconstruction of FXR Beam Conditions," Proceedings of 2001 IEEE Particle Accelerator Conference (2001)

[7] G.P. LeSage, "Time-resolved emittance characterization of an induction linac beam using Optical Transition Radiation," UCRL-ID-153254, November 5, 2002

[8] V.L. Ginsburg and I.M. Frank, JETP (USSR) 16 (1946), 15

[9] J.D. Jackson, Classical Electrodynamics, Wiley (New York, 1975), 685-93

[10] M.L. Ter-Mikaelian, High-Energy Electromagnetic Processes in Condensed Media, WileyInterscience (New York, 1972), 207

[11] V.L. Ginzburg and V.N. Tsytovich, Phys. Rep. 49 (1979) 1

[12] V.E. Pafomov, "Radiation of a charged particle in the presence of a separating boundary" in proceedings of P.N. Lebedev Physics Institute Vol. 44, D.V. Skolbel'tsyn ed. (Consultants Bureau, New York, 1971) 25-157

[13] L. Wartski et. al., Journal of Applied Physics 46 (1975) 3644

[14] R. B. Fiorito and D.W. Rule, "Optical transition radiation beam emittance diagnostics," in Beam Instrumentation Workshop, ed. R.E. Shafer (AIP Conf. Proc. No. 319, AIP Press, 1994).

[15] D.W. Rule, "Transition radiation diagnostics for intense charged particle beams," Nucl. Inst. Meth. B24/25, 901 (1987) 
[16] G.P. LeSage, T.E. Cowan, R.B. Fiorito, D.W. Rule, "Transverse phase space mapping of relativistic electron beams using Optical Transition Radiation," Phys. Rev. ST Accel. Beams 2, December 1999

[17] C. Vermare and D.C. Moir, "Angular Distribution Measurement of an Intense Pulsed Electron Beam using Optical Transition Radiation,” LA-UR-01-1171, March 6, 2001

[18] B. Gitter, "Optical Transition Radiation," 1992, Technical Note online at http://pbpl.physics.ucla.edu/Literature

[19] B.M van Oerle, "A High Brightness Electron Beam for Free Electron Lasers", ISBN 90365 09491

[20] A. Paul, "Flash X-Ray Injector and Accelerator Study”, UCRL-TR-212069, May 6, 2005

[21] Y. Chen, "Final Focus Spot Size in a Solenoid Focusing System," UCRL-ID-152620, April 8,2003

[22] M. Reiser, Theory and Design of Charged Particle Beams, John Wiley \& Sons, Inc. (New York, 1994)

[23] C. Ekdahl, "DAHRT-II Emittance Measurements using Optical Transition Radiation," documentation included with OTR Explorer software

[24] T. Houck, D. Blackfield, J Burke, Y-J Chen, J. Javedani, A. Paul, "Recent Flash X-Ray Injector Modeling”, UCRL-TR-208574, December 14, 2004

[25] A. Paul, "Flash X-Ray Injector and Accelerator Study", UCRL-TR-212069, May 6, 2005 